\title{
Neoclassical equilibria as starting point for global gyrokinetic microturbulence simulations
}

\author{
T. Vernay, ${ }^{1}$ S. Brunner, ${ }^{1}$ L. Villard, ${ }^{1}$ B. F. McMillan, ${ }^{1}$ S. Jolliet, ${ }^{2}$ T. M. Tran, ${ }^{1}$ A. Bottino, ${ }^{3}$ \\ and J. P. Graves ${ }^{1}$ \\ ${ }^{1}$ Centre de Recherches en Physique des Plasmas, Ecole Polytechnique Fédérale de Lausanne, \\ Association EURATOM-Confédération Suisse, CH-1015 Lausanne, Switzerland \\ ${ }^{2}$ Japan Atomic Energy Agency, Higashi-Ueno 6-9-3, Taitou, Tokyo 110-0015, Japan \\ ${ }^{3}$ Max-Planck-Institut für Plasmaphysik, EURATOM Association, Boltzmannstrasse 2, D-85748 Garching, \\ Germany
}

(Received 16 August 2010; accepted 3 November 2010; published online 1 December 2010)

\begin{abstract}
The implementation of linearized operators describing inter- and like-species collisions in the global gyrokinetic particle-in-cell code ORB5 [S. Jolliet, Comput. Phys. Commun. 177, 409 (2007)] is presented. A neoclassical axisymmetric equilibrium with self-consistent electric field can be obtained with no assumption made on the radial width of the particle trajectories. The formulation thus makes it possible to study collisional transport in regions where the neoclassical approximation breaks down such as near the magnetic axis. The numerical model is validated against both analytical results as well as other simulation codes. The effects of the poloidally asymmetric Fourier modes of the electric field are discussed, and the contribution of collisional kinetic electrons is studied. In view of subsequent gyrokinetic simulations of turbulence started from a neoclassical equilibrium, the problem of numerical noise inherent to the particle-in-cell approach is addressed. A novel algorithm for collisional gyrokinetic simulation switching between a local and a canonical Maxwellian background for, respectively, carrying out the collisional and collisionless dynamics is proposed, and its beneficial effects together with a coarse graining procedure [Y. Chen and S. E. Parker, Phys. Plasmas 14, 082301 (2007)] on noise and weight spreading reduction are discussed. (C) 2010 American Institute of Physics. [doi:10.1063/1.3519513]
\end{abstract}

\section{INTRODUCTION}

Global gyrokinetic particle-in-cell (PIC) simulations have been widely recognized as powerful tools for studying microinstabilities and anomalous transport in tokamaks. In the core of such systems, collisionality is weak, and collisionless models have been extensively used. A finite dissipation is nonetheless necessary in order for the nonlinear turbulent system to reach steady state. ${ }^{1,2}$ In a simulation code, this dissipation can be of numerical origin, e.g., related to the finite difference scheme used in Eulerian codes ${ }^{3}$ or produced by an artificial decay of the weights in $\delta f$ PIC Lagrangian codes. ${ }^{4}$ Implementing realistic collision operators enables, however, to account for true physical dissipation, ${ }^{5}$ with interesting effects on microinstability dispersion and associated turbulent transport. Indeed, this so-called anomalous transport, which may lead to particle and heat fluxes much higher than the purely collisional transport predicted by the neoclassical theory, can itself be strongly affected by collisional effects: in particular, in the ion temperature gradient regime, the turbulence level is regulated by the axisymmetric modes, called zonal flows. ${ }^{6}$ Since the zonal flow is damped by collisions, taking the collisional physics into account may lead to a different transport level with respect to collisionless turbulence simulations. ${ }^{7}$ Also, pitch angle scattering may have electrons skip from a trapped to an untrapped trajectory and in this way affect the mechanism of the trapped electron mode (TEM), in particular its growth rate.

In this paper, we present a practical discretization of lin- earized collision operators implemented in the global gyrokinetic PIC code ORB5, ${ }^{8}$ which, in particular, avoids numerical overshoots at the borders of the pitch angle space. There is a computational price for implementing collisions into a gyrokinetic code, especially in the frame of the $\delta f$ PIC approach. The practical implementation of collisions ${ }^{9-12}$ indeed leads to a spreading of weights ${ }^{13}$ with the potential effect of increased numerical noise. Contrary to collisionless simulations, weights thus appear as a growing additional dimension of the numerical system, which, despite spread reduction schemes, ${ }^{13,14}$ requires a larger number of numerical particles to resolve. Furthermore, since the phase-space volumes are not constant in time, a second weight for each marker ${ }^{15}$ is required. Moreover, an algorithm that switches between a local Maxwellian background for carrying out the collisional dynamics and a canonical Maxwellian background for carrying out the collisionless dynamics is proposed. Its positive effects together with a coarse graining procedure ${ }^{14}$ on the noise level of the simulation are pointed out, enabling to start a relevant turbulent simulation from a low-noise neoclassical equilibrium.

The implementation of collision operators in global drift- or gyrokinetic codes including full guiding center trajectories opens many possibilities of investigating issues related to neoclassical transport, which are not reachable with collisionless simulations. Several studies of neoclassical transport have been performed in the past by codes designed specifically for carrying out computations within the neoclassical approximations, ${ }^{16-18}$ i.e., in particular, $\rho_{b} / L_{c} \ll 1$, where 
$\rho_{b}$ is the banana width and $L_{c}$ is the characteristic gradient length of the equilibrium profiles. Going beyond these approximations is clearly of interest. Various studies of neoclassical transport and equilibria using global codes accounting for finite banana width effects have thus already been performed, both considering Lagrangian ${ }^{12,19-21}$ and Eulerian $^{22,23}$ methods. The corresponding axisymmetric electric field ensuring ambipolarity has also been investigated, ${ }^{19-21}$ showing its crucial effect on the neoclassical equilibrium. Such global neoclassical simulations including full ion and electron dynamics will be studied.

This paper is organized as follows: in Sec. II, the simulation model used by ORB5 is briefly explained, including the considered magnetic equilibrium, density and temperature profiles, as well as some definitions related to neoclassical transport. The ORB5 numerical methods are developed in Sec. III, with details concerning the newly implemented collision operators. Neoclassical transport simulation results are presented in Sec. IV, with insights beyond the neoclassical approximation, in particular, simulations including terms neglected within the neoclassical limit such as the effects of the poloidally asymmetric modes. Global simulations including the full kinetic ion and electron dynamics are also studied in the frame of neoclassical runs. Finally, Sec. V discusses the possibility to start gyrokinetic turbulent simulations from a neoclassical equilibrium, focusing particularly on the crucial noise problem. In particular, Sec. V proposes an algorithm enabling collisional runs that take advantage of the collisionless stationary solutions of the gyrokinetic equation. Conclusions are drawn in Sec. VI.

\section{SIMULATION MODEL}

The physical model we consider is the standard gyrokinetic equation within the electrostatic approximation, as derived by Hahm in Ref. 24, to which collisional effects have been added in the form of approximate Landau collision operators. Note that although Hahm's equations have been originally derived under the assumption that $e \phi / T \ll 1$, where $\phi$ is the electrostatic potential and $T$ is the temperature, it has been shown in Ref. 25 that these same equations are, in fact, valid as long as $\left|v_{E \times B} / v_{\mathrm{th}}\right| \sim k_{\perp} \rho_{L} e \phi / T \ll 1$, where $k_{\perp}$ is the perpendicular wave number of the potential and $\rho_{L}$ is the Larmor radius. This subsonic condition is clearly verified for all the neoclassical simulation results presented in this paper.

\section{A. Gyrokinetic equations}

In the frame of the gyrokinetic theory, each species is characterized by its gyroaveraged particle distribution function $f$. The distribution is usually expressed in terms of the gyrocenter variables $\left(\vec{R}, v_{\|}, \mu\right)$, where $\vec{R}$ is the guiding center position, $v_{\|}=\vec{v} \cdot \vec{B} / B$ is the parallel velocity of the particle, $\vec{B}$ is the magnetic field, and $\mu=m v_{\perp}^{2} / 2 B$ is the magnetic moment. The gyrokinetic equation with collisions, governing the evolution of $f$, reads as

$$
\frac{D}{D t} f=-C(f),
$$

where $C$ is the nonlinear Landau collision operator on $f$ and $D / D t$ is the collisionless gyrokinetic operator,

$$
\frac{D}{D t}=\frac{\partial}{\partial t}+\frac{\mathrm{d} \vec{R}}{\mathrm{~d} t} \cdot \frac{\partial}{\partial \vec{R}}+\frac{\mathrm{d} v_{\|}}{\mathrm{d} t} \frac{\partial}{\partial v_{\|}} .
$$

In the limit of electrostatic fluctuations, the time derivative of the gyrocenter variables $\left(\mathrm{d} \vec{R} / \mathrm{d} t, \mathrm{~d} v_{\|} / \mathrm{d} t, \mathrm{~d} \mu / \mathrm{d} t\right)$ implemented as particle trajectories in ORB5 (Ref. 8) are the equations derived by Hahm, ${ }^{24}$

$$
\begin{aligned}
& \frac{\mathrm{d} \vec{R}}{\mathrm{dt}}=\vec{v}_{G}=v_{\|} \hat{b}+\underbrace{\vec{v}_{\nabla B}+\vec{v}_{c}+\vec{v}_{E \times B}}_{\vec{v}_{d}}, \\
& \frac{\mathrm{d} v_{\|}}{\mathrm{dt}}=\frac{1}{m v_{\|}} \vec{v}_{G} \cdot(q \vec{E}-\mu \vec{\nabla} B), \\
& \frac{\mathrm{d} \mu}{\mathrm{dt}}=0,
\end{aligned}
$$

where $m$ and $q$ are the particle mass and charge, respectively, and $\hat{b}=\vec{B} / B$. The drift velocity $\vec{v}_{d}$ is composed of the $\nabla B$ drift $\vec{v}_{\nabla B}$, the curvature drift $\vec{v}_{c}$, and the $\vec{E} \times \vec{B}$ drift $\vec{v}_{E \times B}$. The detailed equations of motion implemented in ORB5 are given in Ref. 8. The collision operator appearing on the right hand side of Eq. (1) is linearized with respect to a local Maxwellian $f_{\mathrm{LM}}: C(f) \approx \hat{C}\left(\delta f_{\mathrm{LM}}\right)$, where $\delta f_{\mathrm{LM}}=f-f_{\mathrm{LM}}$. This approximation is justified as long as $\delta f_{\mathrm{LM}}$ remains small compared to $f_{\mathrm{LM}}$. This statement is clearly verified for the simulation results presented in this paper since the time scale we consider, typically a collision time, is small compared to the neoclassical confinement time. Indeed, an estimate for the deviation from the background distribution is given by $\delta f_{\mathrm{LM}} \sim \Delta r \cdot \nabla f_{\mathrm{LM}} \sim f_{\mathrm{LM}} \Delta r / L_{c}$, where $\Delta r$ is the typical radial excursion of the particle along its collisional trajectory and $L_{c}$ is the characteristic gradient length of the equilibrium profiles. As $\left|\Delta r / L_{c}\right| \ll 1$ after a few collision times for the systems of interest, the scaling $\left|\delta f_{\mathrm{LM}} / f_{\mathrm{LM}}\right| \ll 1$ is indeed ensured. The local Maxwellian background $f_{\mathrm{LM}}$, given in the gyrocenter variables, reads as

$$
f_{\mathrm{LM}}=\frac{n_{0}(\Psi)}{\left[2 \pi T_{0}(\Psi) / m\right]^{3 / 2}} \exp \left[-\frac{m v_{\|}^{2}}{2 T_{0}(\Psi)}-\frac{B \mu}{T_{0}(\Psi)}\right],
$$

where temperature and density are functions of the poloidal magnetic flux $\Psi$. Thus, Eq. (1) reads as

$$
\frac{D}{D t} f=-\hat{C}\left(\delta f_{\mathrm{LM}}\right)
$$

More details are given in Sec. II B. In the neoclassical ordering, i.e., $\left|v_{d} / v_{\|}\right| \sim \rho_{b} / L_{c} \ll 1$, drifts are neglected in the gyrokinetic operator $D / D t$ applied to $\delta f_{\mathrm{LM}}$ so that one considers the following equation instead: 


$$
\left.\frac{D}{D t}\right|_{\text {neo }} \delta f_{\mathrm{LM}}=-\left[\frac{D}{D t} f_{\mathrm{LM}}+\hat{C}\left(\delta f_{\mathrm{LM}}\right)\right]
$$

where

$$
\left.\frac{D}{D t}\right|_{\text {neo }}=\frac{\partial}{\partial t}+v_{\|} \hat{b} \cdot \frac{\partial}{\partial \vec{R}}+\frac{\mathrm{d} v_{\|}}{\mathrm{d} t} \frac{\partial}{\partial v_{\|}} .
$$

The equations for the characteristics underlying the advection operator (9) are thus

$$
\begin{aligned}
& \frac{\mathrm{d} \vec{R}}{\mathrm{dt}}=\vec{v}_{G}=v_{\|} \hat{b}, \\
& \frac{\mathrm{d} v_{\|}}{\mathrm{dt}}=\frac{1}{m} \hat{b} \cdot(q \vec{E}-\mu \vec{\nabla} B), \\
& \frac{\mathrm{d} \mu}{\mathrm{dt}}=0 .
\end{aligned}
$$

Equation (8), which treats drifts perturbatively by keeping the term $\vec{v}_{d}$ only on the right hand side (RHS), will be referred to as the neoclassical limit case. Note that with respect to $\delta f_{\mathrm{LM}}$, this equation is local to a magnetic surface. Conversely, Eq. (7), which treats the finite orbit width effects resulting from drifts $\vec{v}_{d}$ without approximations, will be referred to as the global case.

Let us point out that the usual neoclassical ordering breaks down near the magnetic axis, as the radial excursion of particles from a flux surface can become comparable to the minor radius. In particular, as stated explicitly in Ref. 26, in this region, the ordering $\left|\vec{v}_{d} \cdot \vec{\nabla} \delta f_{\mathrm{LM}}\right| \sim\left|\vec{v}_{d} \cdot \vec{\nabla} f_{\mathrm{LM}}\right|$ applies instead of the standard neoclassical ordering $\left|\vec{v}_{d} \cdot \vec{\nabla} \delta f_{\mathrm{LM}}\right|$ $\ll\left|\vec{v}_{d} \cdot \vec{\nabla} f_{\mathrm{LM}}\right|$, which, in fact, leads to transport being radially nonlocal. It is to be emphasized, however, that this nonlocal ordering does not alter the scaling $\left|\delta f_{\mathrm{LM}}\right| \ll f_{\mathrm{LM}}$. Indeed, it is the gradient and not $\delta f_{\mathrm{LM}}$ itself that becomes large. Considering the term $\vec{v}_{d} \cdot \vec{\nabla} \delta f_{\mathrm{LM}}$ beyond the standard neoclassical theory, along with a linearized collision operator, thus brings a relevant correction to the local approximation breaking down near the axis. Note that this correction does not improve the accuracy of the calculation away from the magnetic axis, since in this case, $\vec{v}_{d} \cdot \vec{\nabla} \delta f_{\mathrm{LM}}$ is of the same neoclassical order as the nonlinear collisionality $C\left[\delta f_{\mathrm{LM}}, \delta f_{\mathrm{LM}}\right]$ we neglect.

The gyrokinetic equation is coupled with the quasineutrality equation. Further considering the decomposition $f_{i}=f_{\mathrm{LM}, i}+\delta f_{\mathrm{LM}, i}$, the background ion density is given by the gyrodensity $\bar{n}_{i 0}$,

$$
\bar{n}_{i 0}(\vec{x})=\int \frac{B_{\|}^{*}}{m} \mathrm{~d}^{3} R \mathrm{~d} v_{\|} \mathrm{d} \mu f_{\mathrm{LM}, i}\left(\vec{R}, v_{\|}, \mu\right) \delta(\vec{R}+\vec{\rho}-\vec{x}),
$$

where $\vec{\rho}(\mu)$ is the Larmor radius vector and $B_{\|}^{*}=B+m v_{\|}$ $\times(\vec{\nabla} \times \hat{b}) \cdot \hat{b} / q$. One assumes that the background densities of electrons and ions verify quasineutrality, i.e., $Z \bar{n}_{i 0}=n_{e 0}$, where $Z$ is the ionization degree. The perturbed ion density has both a perturbed gyrodensity contribution $\overline{\delta n}_{i}$ as well as a polarization drift term $\delta n_{\mathrm{pol}, i}$,

$$
\delta n_{i}(\vec{x})=\overline{\delta n_{i}}+\delta n_{\mathrm{pol}, i} .
$$

In ORB5, the polarization drift term $\delta n_{\mathrm{pol}, i}$ is both linearized and expanded to second order in $k_{\perp} \rho_{L i}$, where $k_{\perp}$ is the characteristic perpendicular wave number and $\rho_{L i}$ is the ion Larmor radius: $\delta n_{\mathrm{pol}, i} \simeq \nabla_{\perp}\left(Z n_{i 0} \nabla_{\perp} \phi / B \Omega_{i}\right)$. Note that the polarization drift linearization assumes that $\left|\delta f_{\mathrm{LM}}\right| \ll f_{\mathrm{LM}}$. In the case of drift-kinetic electrons, the following quasineutrality equation for the fluctuating components is considered:

$$
-\nabla_{\perp} \cdot\left[\frac{Z n_{i 0}(\Psi)}{B \Omega_{i}} \nabla_{\perp} \phi\right]=Z \overline{\delta n_{i}}(\vec{x})-\delta n_{e}(\vec{x})
$$

where $\delta n_{e}$ is the perturbed electron density, $\Omega_{i}$ is the ion cyclotron frequency, and $\phi$ is the electrostatic potential. Since the electrons are considered drift-kinetic in Eq. (15), the electron density is equal to the electron guiding center density. In case an adiabatic electron response may be assumed, the quasineutrality equation for the fluctuating components is instead given by

$$
\begin{aligned}
& \frac{e n_{i 0}(\Psi)}{T_{e}(\Psi)}[\phi(\vec{x}, t)-\langle\phi\rangle(\Psi, t)]-\nabla_{\perp} \cdot\left[\frac{n_{i 0}(\Psi)}{B \Omega_{i}} \nabla_{\perp} \phi\right] \\
& \quad=\overline{\delta n_{i}}(\vec{x}),
\end{aligned}
$$

where $T_{e}$ is the electron temperature, $e$ is the elementary charge, and \langle\rangle stands for the flux surface average operator. Note that the considered electron adiabatic response is linearized, having further invoked $\left|e(\phi-\langle\phi\rangle) / T_{e}\right| \ll 1$.

\section{B. Collision operators}

The finite Larmor radius (FLR) effects resulting from the change of variables from particle to gyrocenter variables, otherwise retained in the collisionless gyrokinetic turbulent dynamics in ORB5, have been neglected with respect to the collision operators. ${ }^{27,28}$ This approximation is justified if the perpendicular wavelength of the electrostatic perturbation is larger than the Larmor radius. This should be well satisfied for all species in the frame of neoclassical transport studies. This assumption should also hold for collisional damping of zonal flows as well as for collisional trapping/detrapping of electrons in the TEM turbulent regime.

\section{Electron-ion collisions}

Invoking the low electron-ion mass ratio, $m_{e} / m_{i} \ll 1$, the electron-ion collisions are modeled by the Lorentz operator, assuming immobile ions in the laboratory frame,

$$
C_{e i}\left[f_{i}, f_{e}\right]=\nu_{e i}(v) \hat{L}^{2} f_{e},
$$

where

$$
\hat{L}^{2}=-\left[\frac{\partial}{\partial \xi}\left(1-\xi^{2}\right) \frac{\partial}{\partial \xi}+\frac{1}{1-\xi^{2}} \frac{\partial^{2}}{\partial \alpha^{2}}\right],
$$

having introduced the pitch angle variable $\xi=v_{\|} / v$ and the gyroangle $\alpha$. Considering the decomposition $f_{e}=f_{\mathrm{LM}, e}$ $+\delta f_{\mathrm{LM}, e}$, one obtains 


$$
C_{e i}\left[f_{i}, f_{e}\right]=C_{e i}\left[f_{i}, \delta f_{\mathrm{LM}, e}\right]=\nu_{e i}(v) \hat{L}^{2} \delta f_{\mathrm{LM}, e},
$$

having noted that $C_{e i}\left[f_{i}, f_{\mathrm{LM}, e}\right]=0$, as the Lorentz operator annihilates any isotropic velocity distribution function. The electron-ion collision frequency $\nu_{e i}(v)$ is given by

$$
\begin{aligned}
& \nu_{e i}(v)=\bar{\nu}_{e i}\left(\frac{v_{\text {th } e}}{v}\right)^{3}, \\
& \bar{\nu}_{e i}=\frac{n_{i} Z^{2} e^{4} \ln \Lambda}{8 \pi \epsilon_{0}^{2} m_{e}^{2} v_{\text {th } e}^{3}},
\end{aligned}
$$

where $m_{e}$ is the electron mass, $v_{\text {th } e}=\sqrt{T_{e} / m_{e}}$ is the electron thermal velocity, $\epsilon_{0}$ is the permittivity of free space, and $\ln \Lambda$ is the Coulomb logarithm. It is assumed that $\ln \Lambda$ is constant over the whole plasma, and one approximates $n_{i} \approx n_{i 0}(\Psi)$ for computing the collision frequency, where $n_{i 0}(\Psi)$ is the density of the local Maxwellian background. The distribution being invariant with respect to $\alpha$ in the gyrokinetic description, the operator $C_{e i}$ thus reduces to

$$
C_{e i}\left[f_{i}, \delta f_{\mathrm{LM}, e}\right]=-\nu_{e i}(v) \frac{\partial}{\partial \xi}\left[\left(1-\xi^{2}\right) \frac{\partial \delta f_{\mathrm{LM}, e}}{\partial \xi}\right] .
$$

\section{Self-collisions}

Self-collisions are treated through a linearized operator and are split into two terms. Considering again a local Maxwellian background $f_{\mathrm{LM}}$, the full distribution is decomposed into $f=f_{\mathrm{LM}}+\delta f_{\mathrm{LM}}$. Using the bilinearity of the collision operator with respect to each of its two arguments, one obtains

$$
\begin{aligned}
C[f, f]= & \underbrace{C\left[f_{\mathrm{LM}}, f_{\mathrm{LM}}\right]}_{0}+C\left[f_{\mathrm{LM}}, \delta f_{\mathrm{LM}}\right]+C\left[\delta f_{\mathrm{LM}}, f_{\mathrm{LM}}\right] \\
& +\underbrace{C\left[\delta f_{\mathrm{LM}}, \delta f_{\mathrm{LM}}\right]}_{\text {neglected }},
\end{aligned}
$$

where the term $C\left[f_{\mathrm{LM}}, f_{\mathrm{LM}}\right]$ cancels out as $f_{\mathrm{LM}}$ is a stationary state of the self-collision operator. The term $C\left[\delta f_{\mathrm{LM}}, \delta f_{\mathrm{LM}}\right]$ is neglected as a result of the linearization process. The linearized self-collision operator thus finally reads as

$$
\hat{C}\left(\delta f_{\mathrm{LM}}\right)=C\left[f_{\mathrm{LM}}, \delta f_{\mathrm{LM}}\right]+C\left[\delta f_{\mathrm{LM}}, f_{\mathrm{LM}}\right] .
$$

The term $C\left[f_{\mathrm{LM}}, \delta f_{\mathrm{LM}}\right]$, corresponding to the collisions of the perturbation $\delta f_{\mathrm{LM}}$ on the local Maxwellian background $f_{\mathrm{LM}}$, is given by

$$
C\left[f_{\mathrm{LM}}, \delta f_{\mathrm{LM}}\right]=\frac{\partial}{\partial \vec{v}} \cdot\left[\vec{\Gamma}(\vec{v}) \delta f_{\mathrm{LM}}-\frac{\partial}{\partial \vec{v}} \cdot \mathbf{D}(\vec{v}) \delta f_{\mathrm{LM}}\right],
$$

where the drag vector $\vec{\Gamma}$ and the diffusion tensor $\mathbf{D}$ are written as follows: ${ }^{13}$

$$
\vec{\Gamma}=-\bar{\nu} H(\mathrm{v}) \vec{v},
$$

$$
\mathbf{D}=\frac{\bar{\nu} v_{\mathrm{th}}^{2}}{4}\left(K(\mathrm{v})\left[\mathbf{I}-\frac{\vec{v}: \vec{v}}{v^{2}}\right]+2 H(\mathrm{v}) \frac{\vec{v}: \vec{v}}{v^{2}}\right)
$$

with $\mathbf{I}$ being the identity tensor and

$$
\bar{\nu}=\frac{n q^{4} \ln \Lambda}{2 \pi \epsilon_{0}^{2} m^{2} v_{\text {th }}^{3}}
$$

being the thermal self-collision frequency for the species with mass $m$, charge $q$, thermal velocity $v_{\text {th }}=\sqrt{T / m}$, and background density $n$. Defining the normalized velocity $v$ $=v / v_{\text {th }}$, the functions $H, K$, and $G$ related to the Rosenbluth potentials are introduced:

$$
\begin{aligned}
& H(\mathrm{v})=\frac{1}{\mathrm{v}^{3}}\left[\operatorname{erf}(\mathrm{v} / \sqrt{2})-\sqrt{\frac{2}{\pi}} \mathrm{v} e^{-\mathrm{v}^{2} / 2}\right], \\
& K(\mathrm{v})=\frac{1}{\mathrm{v}^{3}}\left[\left(\mathrm{v}^{2}-1\right) \operatorname{erf}(\mathrm{v} / \sqrt{2})+\sqrt{\frac{2}{\pi}} \mathrm{v}^{-\mathrm{v}^{2} / 2}\right], \\
& G(\mathrm{v})=\left(2 \mathrm{v}^{2}-1\right) H(\mathrm{v})-K(\mathrm{v}),
\end{aligned}
$$

using the definition $\operatorname{erf}(x)=(2 / \sqrt{\pi}) \int_{0}^{x} \mathrm{~d} t \exp \left(-t^{2}\right)$ for the error function. The operator $C\left[f_{\mathrm{LM}}, \delta f_{\mathrm{LM}}\right]$ given by Eq. (24) can, in fact, be explicitly decomposed into a pitch angle scattering term and a thermalization term,

$$
\begin{aligned}
C\left[f_{\mathrm{LM}}, \delta f_{\mathrm{LM}}\right]= & \frac{\nu_{D}(v)}{2} \hat{L}^{2} \delta f_{\mathrm{LM}} \\
& -\frac{1}{v^{2}} \frac{\partial}{\partial v}\left[\frac{v^{4}}{2} \nu_{\|}(v) f_{\mathrm{LM}} \frac{\partial}{\partial v}\left(\frac{\delta f_{\mathrm{LM}}}{f_{\mathrm{LM}}}\right)\right],
\end{aligned}
$$

having defined $\nu_{D}(v)=\bar{\nu} K(\mathrm{v}) / 2 \mathrm{v}^{2}$ as the pitch angle frequency and $\nu_{\|}(v)=\bar{\nu} H(\mathrm{v}) / \mathrm{v}^{2}$ as the thermalization frequency.

The term $C\left[\delta f_{\mathrm{LM}}, f_{\mathrm{LM}}\right]$ is the background reaction that ensures conservation of mass, momentum, and kinetic energy of the linearized self-collision operator. For practical reasons, to avoid having to reconstruct $\delta f_{\mathrm{LM}}$ and taking its derivative at each time step, operations strongly subject to numerical noise in a $\delta f$ PIC code, one does not implement the exact form of this operator, as derived directly from the Landau operator, but makes use of an approximate form $C\left[\delta f_{\mathrm{LM}}, f_{\mathrm{LM}}\right] \simeq f_{\mathrm{LM}} \mathcal{B}\left(\delta f_{\mathrm{LM}}\right),{ }^{12,13}$

$$
\begin{aligned}
\frac{C\left[\delta f_{\mathrm{LM}}, f_{\mathrm{LM}}\right]}{f_{\mathrm{LM}}} & \simeq \mathcal{B}\left(\delta f_{\mathrm{LM}}\right) \\
& =\frac{1}{n(\vec{x})}\left\{6 \sqrt{\pi} H(\mathrm{v}) \frac{\delta \mathcal{P}_{\|} v_{\|}}{v_{\mathrm{th}}^{2}}+\sqrt{\pi} G(\mathrm{v}) \frac{\delta \mathcal{E}}{v_{\mathrm{th}}^{2}}\right\},
\end{aligned}
$$

where $\delta \mathcal{P}_{\|}$and $\delta \mathcal{E}$ are the changes in the parallel momentum and the kinetic energy of the perturbation distribution, respectively, due to the operator defined in Eq. (24),

$$
\delta P_{\|}\left(\delta f_{\mathrm{LM}}, \vec{x}\right)=-\int C\left[f_{\mathrm{LM}}, \delta f_{\mathrm{LM}}\right] v_{\|} \mathrm{d}^{3} v,
$$




$$
\delta \mathcal{E}\left(\delta f_{\mathrm{LM}}, \vec{x}\right)=-\int C\left[f_{\mathrm{LM}}, \delta f_{\mathrm{LM}}\right] v^{2} \mathrm{~d}^{3} v .
$$

Let us notice that due to the invariance of the distribution function with respect to the gyroangle, we only need to consider the variation of the parallel component of the velocity in Eq. (33). The operator $\mathcal{B}$, although a physical simplification, nevertheless satisfies the local conservation properties. The functional form of Eq. (32) indeed ensures that

$$
\begin{aligned}
& \int f_{\mathrm{LM}} \mathcal{B}\left(\delta f_{\mathrm{LM}}\right) \mathrm{d}^{3} v=0, \\
& \int f_{\mathrm{LM}} \mathcal{B}\left(\delta f_{\mathrm{LM}}\right) v_{\|} \mathrm{d}^{3} v+\delta \mathcal{P}_{\|}\left(\delta f_{\mathrm{LM}}, \vec{x}\right)=0, \\
& \int f_{\mathrm{LM}} \mathcal{B}\left(\delta f_{\mathrm{LM}}\right) v^{2} \mathrm{~d}^{3} v+\delta \mathcal{E}\left(\delta f_{\mathrm{LM}}, \vec{x}\right)=0 .
\end{aligned}
$$

The operators $C\left[f_{\mathrm{LM}}, \delta f_{\mathrm{LM}}\right]$ and $f_{\mathrm{LM}} \mathcal{B}\left(\delta f_{\mathrm{LM}}\right)$ thus together conserve parallel momentum and kinetic energy and individually conserve mass. One can furthermore show ${ }^{12,13}$ that the operators (24) and (32) verify properties of selfadjointness, satisfy the $\mathrm{H}$-theorem, and that a linearized shifted Maxwellian of the form

$$
\delta f_{\mathrm{LM}}=f_{\mathrm{LM}}\left(c_{1}+c_{2} \frac{v_{\|}}{v_{\mathrm{th}}}+c_{3} \frac{v^{2}}{v_{\mathrm{th}}^{2}}\right)
$$

will be annihilated by the total collision operator, i.e., $C\left[f_{\mathrm{LM}}, \delta f_{\mathrm{LM}}\right]+f_{\mathrm{LM}} \mathcal{B}\left(\delta f_{\mathrm{LM}}\right)=0$ for any $\delta f_{\mathrm{LM}}$ of the form (38), where $c_{1}, c_{2}$, and $c_{3}$ are arbitrary constants.

\section{Equilibrium profiles}

The considered "ad hoc" equilibrium ${ }^{29}$ consists of toroidal, axisymmetric, nested magnetic surfaces with circular, concentric, and poloidal cross sections. In this case, the axisymmetric magnetic field is given by $\vec{B}=\vec{\nabla} \Psi \times \vec{\nabla} \varphi$ $+F(\Psi) \vec{\nabla} \varphi$, assuming that $\Psi=\Psi(r)$ with $\mathrm{d} \Psi / \mathrm{d} r=r B_{0} / \bar{q}$, as well as $F=R_{0} B_{0}$, so that

$$
\vec{B}=\frac{B_{0} R_{0}}{R}\left[\hat{e}_{\varphi}+\frac{r}{R_{0} \bar{q}(r)} \hat{e}_{\theta}\right],
$$

where $R$ is the major radius, $r$ is the local minor radius, $\varphi$ is the toroidal angle, $\theta$ is the poloidal angle, and $\hat{e}_{\varphi}$ and $\hat{e}_{\theta}$ are the unitary vectors in the toroidal and poloidal directions, respectively. Furthermore, $B_{0}$ and $R_{0}$ stand for the magnetic field amplitude and major radius on the magnetic axis $(r=0)$. The profile $\bar{q}$ is chosen quadratic, introducing the global minor radius $a$,

$$
\bar{q}(r)=\bar{q}_{0}+\bar{q}_{1} \frac{r^{2}}{a^{2}},
$$

which is related to the safety factor profile $q_{s}(r)$ by the following relation:

$$
\bar{q}(r)=\sqrt{1-\epsilon^{2}} q_{s}(r),
$$

where $\epsilon=r / R_{0}$ is the local inverse aspect ratio. For all simulation results presented in Secs. IV and $\mathrm{V}$, the values $\bar{q}_{0}$ $=0.854$ and $\bar{q}_{1}=2.184$ have been chosen .

In this paper, we use several kinds of temperature and density profiles. Let $\mathcal{A}$ stand for either the temperature $T$ or density $n$ of a given species. The first kind of profile, referred to as type 1 , is defined with respect to the poloidal flux coordinate $s=\sqrt{\Psi / \Psi_{\text {edge }}}$,

$$
\begin{aligned}
\frac{\mathrm{d} \ln \mathcal{A}}{\mathrm{d} s^{2}}= & -\frac{\kappa_{\mathcal{A}}}{\left[1-\cosh ^{-2}\left(s_{0} / \Delta_{\mathcal{A}}\right)\right]} \\
& \times\left[\cosh ^{-2}\left(\frac{s-s_{0}}{\Delta_{\mathcal{A}}}\right)-\cosh ^{-2}\left(\frac{s_{0}}{\Delta_{\mathcal{A}}}\right)\right],
\end{aligned}
$$

where $s_{0}=0.5$ is chosen so that $\mathrm{d} \ln \mathcal{A} / \mathrm{d} s^{2}=0$ for $s=0,1$ and $\mathrm{d} \ln \mathcal{A} / \mathrm{d} s^{2}=-\kappa_{\mathcal{A}}$ for $s=s_{0}$. Profiles referred to as type 2 are defined with respect to the geometrical radial coordinate $r$, with a similarly peaked shape as profiles of type 1,

$$
\frac{\mathrm{d} \ln \mathcal{A}}{\mathrm{d}(r / a)}=-\kappa_{\mathcal{A}} \cosh ^{-2}\left(\frac{r / a-\widetilde{r}_{0}}{\Delta_{\mathcal{A}}}\right),
$$

with $\widetilde{r}_{0}=0.5$. Profiles referred to as type 3 are defined with respect to the same coordinate $r$, with a flat logarithmic gradient over a wide region of the torus,

$$
\begin{aligned}
\frac{\mathrm{d} \ln \mathcal{A}}{\mathrm{d}(r / a)}= & -\frac{\kappa_{\mathcal{A}}}{2}\left\{\tanh \left[\frac{r / a-\left(\widetilde{r}_{0}-\Delta_{\mathcal{A}}\right)}{\Delta_{r}}\right]\right. \\
& \left.-\tanh \left[\frac{r / a-\left(\widetilde{r}_{0}+\Delta_{\mathcal{A}}\right)}{\Delta_{r}}\right]\right\},
\end{aligned}
$$

where the values $\widetilde{r}_{0}=0.5, \Delta_{r}=0.04$, and $\Delta_{\mathcal{A}}=0.4$ have been chosen.

\section{Fluxes, diffusivity, and force balance}

We define, respectively, the particle flux $\Gamma$, the kinetic energy flux $Q_{\text {kin }}$, the potential energy flux $Q_{\text {pot }}$, the heat flux $q_{H}$, and the bootstrap current $j_{b}$ for each species as follows:

$$
\begin{aligned}
& \Gamma=\left\langle\frac{\vec{\nabla} \Psi}{|\vec{\nabla} \Psi|} \cdot \int \mathrm{d}^{3} v f \frac{\mathrm{d} \vec{R}}{\mathrm{~d} t}\right\rangle_{S}=\left\langle\frac{1}{|\vec{\nabla} \Psi|} \int \mathrm{d}^{3} v f \frac{\mathrm{d} \Psi}{\mathrm{d} t}\right\rangle_{S}, \\
& Q_{\text {kin }}=\left\langle\frac{1}{|\vec{\nabla} \Psi|} \int \mathrm{d}^{3} v \frac{m}{2} v^{2} f \frac{\mathrm{d} \Psi}{\mathrm{d} t}\right\rangle_{S} \\
& Q_{\text {pot }}=\left\langle\frac{1}{|\vec{\nabla} \Psi|} \int \mathrm{d}^{3} v q \phi f \frac{\mathrm{d} \Psi}{\mathrm{d} t}\right\rangle_{S}, \\
& q_{H}=\left\langle\frac{1}{|\vec{\nabla} \Psi|} \int \mathrm{d}^{3} v f\left[\frac{m}{2}\left(v^{2}-5 v_{\mathrm{th}}^{2}\right)+q \phi\right] \frac{\mathrm{d} \Psi}{\mathrm{d} t}\right\rangle_{S},
\end{aligned}
$$




$$
j_{b}=\left\langle B \int \mathrm{d}^{3} v f v_{\|}\right\rangle_{\Delta V},
$$

where $\mathrm{d} \Psi / \mathrm{d} t=\vec{\nabla} \Psi \cdot \mathrm{d} \vec{R} / \mathrm{d} t$ and $\langle\mathcal{A}\rangle_{S}=(1 / S) \int_{S} \mathcal{A} \mathrm{d} \sigma$ is defined as the poloidal flux surface average of a quantity $\mathcal{A}$, with $S$ being the surface value. $\langle\mathcal{A}\rangle_{\Delta V}=\int_{\Delta V} \mathcal{A d}{ }^{3} x / \Delta V$ stands for the volume average over the small volume $\Delta V$ enclosed between two neighboring magnetic surfaces $\Psi$ and $\Psi+\Delta \Psi$. In practice, to ensure sufficient statistical accuracy from the PIC approach, surface averages are replaced by volume averages using the relation $\langle\mathcal{A}\rangle_{S}=\langle|\vec{\nabla} \Psi| \mathcal{A}\rangle_{\Delta V} /\langle|\vec{\nabla} \Psi|\rangle_{\Delta V}$. The heat flux can be written as $q_{H}=Q_{\mathrm{kin}}+Q_{\mathrm{pot}}-\frac{5}{2} T \Gamma$. Accounting for the contribution $Q_{\text {pot }}$ from the potential energy $q \phi$ when computing the heat flux $q_{H}$ is essential, as first pointed out by Helander in Ref. 30. Neglecting this term leads to unphysically large contribution from $\vec{v}_{E \times B}$ to the heat flux in the presence of nonzero poloidal mode numbers. For diagnostic purposes, the particle and heat diffusivities are estimated in certain cases using the following relations, respectively, $D=\Gamma /|\nabla n|$ and $\chi_{H}=q_{H} /(n|\nabla T|)$. Let us emphasize that these relations are based on the approximation of neglecting the off-diagonal elements of the transport matrix. In general, temperature gradients contribute also to the particle flux, in the same way as the density gradient contributes to the heat flux. Diffusivities are usually normalized with respect to the gyro-Bohm (GB) units: $\chi_{\mathrm{GB}}=c_{s} \rho_{s}^{2} / a$, where $c_{s}$ is the sound speed, $c_{s}=\sqrt{Z T_{e} / m_{i}}$, and the sound Larmor radius is defined as $\rho_{s}=c_{s} / \Omega_{i}$. The size of the considered plasma is given by the parameter $\rho^{*}=\rho_{s} / a$. For typical simulations presented in this paper, $\rho^{*} \sim 1 / 100$. Note that assuming that $T_{e}=T_{i}, Z=1$, and $a \sim L_{n} \sim L_{T}$, the neoclassical assumption $\rho_{b} / L_{T} \sim \rho_{b} / L_{n}$ $\sim \rho^{*} q_{s} / \sqrt{\epsilon} \ll 1$ is still valid in a large part of the plasma.

An important neoclassical equation is the ion force balance, which reflects the conservation of the toroidal momentum,

$$
\frac{j_{b i}}{n_{i}} \frac{\mathrm{d} \Psi}{\mathrm{d} r}=\frac{R B_{\varphi} T_{i}}{Z e}\left[(k-1) \frac{\mathrm{d}}{\mathrm{d} r} \ln T_{i}-\frac{\mathrm{d}}{\mathrm{d} r} \ln n_{i}+\frac{Z e E_{r}}{T_{i}}\right],
$$

where $E_{r}$ is the radial electric field, $n_{i}$ is the ion density, and $j_{b i}$ is the ion contribution to the bootstrap current. This relation is derived by Hinton and Hazeltine in Ref. 31 [Eq. (6.134)], but where a ratio $B_{\varphi} / B_{\text {poloidal }}$ appears to be missing. The coefficient $k$ predicted by ORB5 will be studied, as well as neoclassical transport coefficients, with respect to the collisionality parameter $\nu^{*}$. Defined basically as the ratio between the detrapping collision frequency and the bounce frequency, it reads for ions as

$$
\nu_{i}^{*}=\frac{R_{0} q_{s}}{\tau_{i i} v_{\text {th } i} \epsilon^{3 / 2}}, \quad \text { with } \tau_{i i}=\frac{6 \sqrt{\pi}}{\nu_{i i}}
$$

and for electrons as

$$
\nu_{e}^{*}=\frac{R_{0} q_{s}}{\tau_{e} v_{\text {th } e} \epsilon^{3 / 2}}, \quad \text { with } \tau_{e}=\frac{3 \sqrt{2 \pi}}{4 \nu_{e i}},
$$

with $\nu_{e i}$ and $\nu_{i i}$ being the collision frequencies as given by Eqs. (20) and (27), respectively.

\section{NUMERICAL METHODS}

The code ORB5 uses a $\delta f$ PIC algorithm, where the full distribution $f$ is decomposed into a numerical background $f_{0}$ and a deviation $\delta f: f=f_{0}+\delta f$. For collisional runs, since the collision operators are linearized with respect to a local Maxwellian (Sec. II B), this latter distribution is an obvious choice for the numerical ion and electron backgrounds: $f_{0}$ $=f_{\text {LM }}$. This particular choice for $f_{0}$ is more obvious in the case of simulations carried out in the standard neoclassical limit, i.e., evolving Eq. (8), which results from a linearization with respect to $f_{\mathrm{LM}}$ of the whole gyrokinetic equation (collisional and collisionless dynamics). The collision operators are treated separately from the collisionless dynamics, according to a time splitting approach.

For the following, let us introduce the notation $D^{\prime} / D t$, which stands for $D / D t$ in the global case and for $D /\left.D t\right|_{\text {neo }}$ in the neoclassical limit, as defined in Sec. II, so that one may always write the gyrokinetic equation (7) or Eq. (8) as follows:

$$
\frac{D^{\prime}}{D t} \delta f_{\mathrm{LM}}=-\left[\frac{D}{D t} f_{\mathrm{LM}}+\hat{C}\left(\delta f_{\mathrm{LM}}\right)\right]
$$

\section{A. Solving the gyrokinetic equation with collisions using the $\delta f$ approach}

Equation (53) for $\delta f_{\mathrm{LM}}$ is solved using the low-noise PIC $\delta f$-method. The linearized collision operator $\hat{C}\left(\delta f_{\mathrm{LM}}\right)$ can, in general, be decomposed into two terms,

$$
\hat{C}\left(\delta f_{\mathrm{LM}}\right)=\underbrace{C_{\mathrm{TP}}\left(\delta f_{\mathrm{LM}}\right)}_{\text {test particle term }}+\underbrace{C_{\mathrm{BR}}\left(\delta f_{\mathrm{LM}}\right)}_{\text {background reaction term }} .
$$

In the case of electrons, for example, $e-i$ collisions contribute to $C_{\mathrm{TP}}$ with the Lorentz operator (19) and $e$-e self-collisions contribute to both $C_{\mathrm{TP}}$ and $C_{\mathrm{BR}}$ with the operators $C\left[f_{\mathrm{LM}}, \delta f_{\mathrm{LM}}\right]$ and $C\left[\delta f_{\mathrm{LM}}, f_{\mathrm{LM}}\right]$ given by Eqs. (24) and (32), respectively. Thus, Eq. (53) for $\delta f_{\mathrm{LM}}$ reads as

$$
\frac{\mathcal{D}}{\mathcal{D} t} \delta f_{\mathrm{LM}}=-\left[\frac{D}{D t} f_{\mathrm{LM}}+C_{\mathrm{BR}}\left(\delta f_{\mathrm{LM}}\right)\right] \text {. }
$$

where the operator $\mathcal{D} / \mathcal{D} t$ is defined as

$$
\mathcal{D} / \mathcal{D} t=D^{\prime} / D t+C_{\mathrm{TP}} .
$$

The PIC representation requires introducing a set of $\mathrm{N}$ socalled numerical markers in the gyrokinetic phase space $z=\left(\vec{R}, v_{\|}, \mu\right)$. Using the notation $z_{r}(t), r=1, \ldots, N$, for the orbits of these markers, the marker distribution function $g(z, t)$ can be written in a discretized form as follows:

$$
g(z, t) \approx \sum_{r=1}^{N} \delta\left[z-z_{r}(t)\right]
$$

The physical distribution $\delta f$ is linked to the marker distribution $g$ by the so-called weight field $W(z, t)$,

$$
\delta f_{\mathrm{LM}}(z, t)=W(z, t) g(z, t),
$$

which in discretized form, using Eq. (57), becomes 


$$
\begin{aligned}
\delta f_{\mathrm{LM}}(z, t) & \approx W(z, t) \sum_{r=1}^{N} \delta\left[z-z_{r}(t)\right] \\
& =\sum_{r=1}^{N} W\left[z_{r}(t), t\right] \delta\left[z-z_{r}(t)\right] \\
& =\sum_{r=1}^{N} w_{r}(t) \delta\left[z-z_{r}(t)\right],
\end{aligned}
$$

having introduced the so-called particle weight $w_{r}(t)$ defined here as the value of the weight field at the marker position,

$$
w_{r}(t):=W\left[z_{r}(t), t\right] .
$$

In the same way, one may introduce the second weight field $P(z, t)$, linking the physical background distribution $f_{\mathrm{LM}}$ to $g,{ }^{15}$

$$
f_{\mathrm{LM}}(z, t)=P(z, t) g(z, t),
$$

which in discretized form, similar to Eq. (59), becomes

$$
f_{\mathrm{LM}}(z, t) \approx \sum_{r=1}^{N} p_{r}(t) \delta\left[z-z_{r}(t)\right]
$$

having introduced the second weight $p_{r}(t)$ of the marker, defined here as

$$
p_{r}(t):=P\left[z_{r}(t), t\right] .
$$

At least formally, there is quite some freedom as to the choice of the evolution of the marker distribution $g$. However, for practical reasons, it is convenient to consider the following equation:

$$
\frac{\mathcal{D}}{\mathcal{D} t} g=0
$$

Using the PIC representation (57), the evolution equation (64) for $g$ is ensured by the marker trajectory equations,

$$
\frac{\delta}{\delta t} z_{r}(t)=\left.\frac{\mathrm{d}}{\mathrm{d} t}\right|_{\text {no coll }} z_{r}(t)+\left.\frac{\delta}{\delta t}\right|_{\text {coll }} z_{r}(t),
$$

where $\mathrm{d} /\left.\mathrm{d} t\right|_{\text {no coll }} z_{r}(t)$ stands for the collisionless part of the trajectories and is given by Eqs. (3)-(5) in the case of global simulations and by Eqs. (10)-(12) in the limit of the neoclassical ordering. The time variation $\delta /\left.\delta t\right|_{\text {coll }} z_{r}(t)$ stands for the collisional contribution accounting for the $C_{\mathrm{TP}}$ operator. The equation of motion (65) clearly represents stochastic trajectories resulting from random velocity kicks, which must be sampled from appropriate probability distribution functions (PDFs) for correctly representing $C_{\mathrm{TP}}$. This type of stochastic equations of motion is called Langevin equations. In ORB5, the collisionless and the collisional dynamics are handled sequentially using a time splitting approach. To represent $\delta f$ with the numerical markers still requires, according to Eq. (59), an equation for the marker weight $w_{r}(t)$. For this purpose, one writes somewhat more explicitly the time derivative along trajectories (65) acting as an operator on a phasespace field,

$$
\frac{\delta}{\delta t}=\frac{D^{\prime}}{D t}+\left(\vec{\Gamma}_{\mathrm{TP}}+\vec{a}_{c}\right) \cdot \frac{\partial}{\partial \vec{v}}
$$

where $\vec{\Gamma}_{\mathrm{TP}}$ is the drag and $\vec{a}_{c}$ is the random acceleration related to the diffusion part of the collision operator $C_{\mathrm{TP}}$, which, in general, can be written as

$$
C_{\mathrm{TP}}\left(\delta f_{\mathrm{LM}}\right)=\frac{\partial}{\partial \vec{v}} \cdot\left[\vec{\Gamma}_{\mathrm{TP}}(\vec{v}) \delta f_{\mathrm{LM}}-\frac{\partial}{\partial \vec{v}} \cdot \mathbf{D}_{\mathrm{TP}}(\vec{v}) \delta f_{\mathrm{LM}}\right] .
$$

The operator $\delta / \delta t$ is related to $\mathcal{D} / \mathcal{D} t$ by the relation

$$
\frac{\delta}{\delta t}=\frac{\mathcal{D}}{\mathcal{D} t}-\left(\frac{\partial}{\partial \vec{v}} \cdot \vec{\Gamma}_{\mathrm{TP}}\right)+\vec{a}_{c} \cdot \frac{\partial}{\partial \vec{v}}+\frac{\partial^{2}}{\partial \vec{v} \partial \vec{v}}: \mathbf{D}_{\mathrm{TP}} .
$$

To obtain an evolution equation for each weight $w_{r}(t)$, one starts by taking its time derivative

$$
\begin{aligned}
\frac{\mathrm{d}}{\mathrm{d} t} w_{r}(t) & =\frac{\delta}{\delta t} W\left[z=z_{r}(t), t\right] \\
& =\frac{\delta}{\delta t}\left(\frac{\delta f_{\mathrm{LM}}}{g}\right)=\frac{1}{g} \frac{\delta}{\delta t} \delta f_{\mathrm{LM}}-\frac{\delta f_{\mathrm{LM}}}{g^{2}} \frac{\delta}{\delta t} g,
\end{aligned}
$$

the last step having been carried out using the chain rule, which is justified as $\delta / \delta t$ represents a first order differential operator. In practice, one proceeds by making use of Eq. (68), but dropping the last two terms on the RHS of this relation. ${ }^{13}$ Thus, one carries on by considering

$$
\frac{\delta}{\delta t} \stackrel{\underline{D}}{=} \frac{\mathcal{D}}{\mathcal{D} t}-\left(\frac{\partial}{\partial \vec{v}} \cdot \vec{\Gamma}_{\mathrm{TP}}\right)
$$

where the notation $\stackrel{!}{=}$ points out what may appear to be an $a d$ hoc simplification. Inserting Eq. (70) into Eq. (69) thus finally leads to

$$
\begin{aligned}
\frac{\mathrm{d}}{\mathrm{d} t} w_{r}(t) & \stackrel{!}{=} \frac{1}{g} \frac{\mathcal{D}}{\mathcal{D} t} \delta f_{\mathrm{LM}}-\frac{\delta f_{\mathrm{LM}}}{g^{2}} \frac{\mathcal{D}}{\mathcal{D} t} g \\
& =-\left.\frac{1}{g}\left[\frac{D}{D t} f_{\mathrm{LM}}+C_{\mathrm{BR}}\left(\delta f_{\mathrm{LM}}\right)\right]\right|_{\left[z_{r}(t), t\right]},
\end{aligned}
$$

having made use of Eqs. (55) and (64) and noticing that the contribution from the compressibility of the drag term, $\partial / \partial \vec{v} \cdot \vec{\Gamma}_{\mathrm{TP}}$, in Eq. (70) cancels out. Contrary to a collisionless system, the value of the marker distribution $g(z, t)$ is not invariant along the marker trajectories $z_{r}(t)$. There is thus, a priori, a practical problem at evaluating the RHS of relation (71), involving the factor $1 / g\left[z_{r}(t), t\right]$, which can be identified to the phase-space volume associated with the marker. This is solved by making use of the second weight $p_{r}(t)=P\left[z_{r}(t), t\right]=f_{\mathrm{LM}} /\left.g\right|_{\left[z_{r}(t), t\right]}$, so Eq. (71) becomes

$$
\frac{\mathrm{d}}{\mathrm{d} t} w_{r}(t)=-\left.p_{r}(t) \frac{1}{f_{\mathrm{LM}}}\left[\frac{D}{D t} f_{\mathrm{LM}}+C_{\mathrm{BR}}\left(\delta f_{\mathrm{LM}}\right)\right]\right|_{\left[z_{r}(t), t\right]} .
$$

The details on evaluating $C_{\mathrm{BR}}$ are discussed in Sec. III D. An equation for the second weight $p_{r}(t)$ is now necessary and is derived in a similar way as for $w_{r}(t)$, 


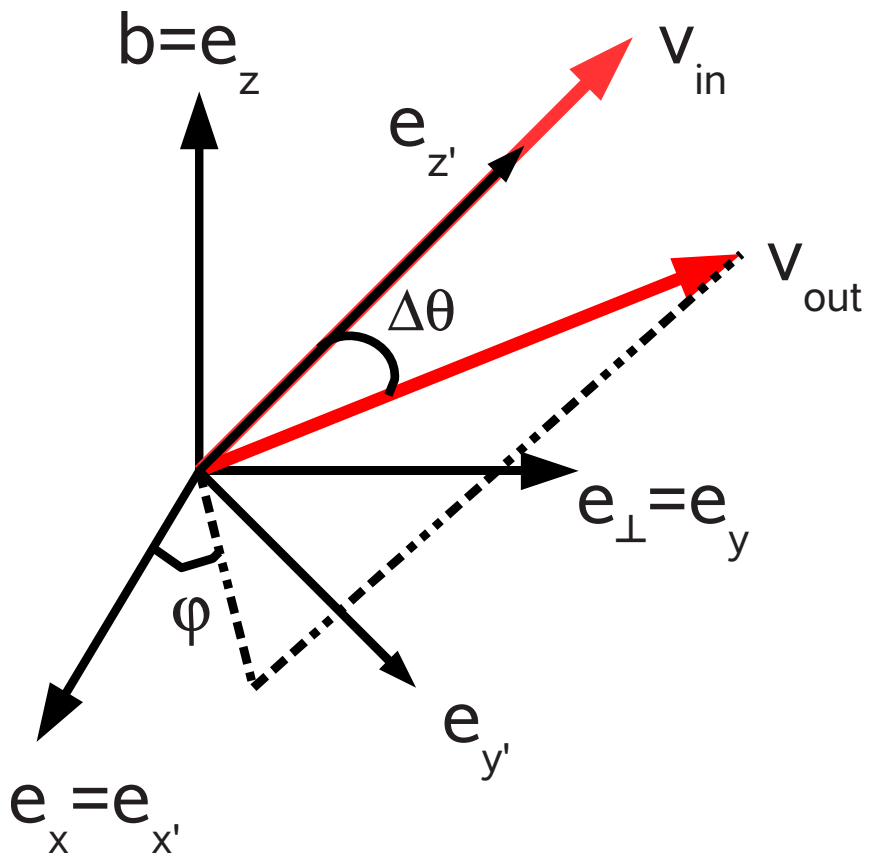

FIG. 1. (Color online) Extended three-dimensional velocity space for random kicks, with the different coordinate systems. The velocities before the kick $\vec{v}_{\text {in }}$ and after the kick $\vec{v}_{\text {out }}$ are shown.

$$
\begin{aligned}
\frac{\mathrm{d}}{\mathrm{d} t} p_{r}(t) & =\frac{\delta}{\delta t} P\left[z=z_{r}(t), t\right] \\
& =\frac{\delta}{\delta t}\left(\frac{f_{\mathrm{LM}}}{g}\right) \\
& =\frac{1}{g} \frac{\delta}{\delta t} f_{\mathrm{LM}}-\frac{f_{\mathrm{LM}}}{g^{2}} \frac{\delta}{\delta t} g \stackrel{!}{=} \frac{1}{g} \frac{\mathcal{D}}{\mathcal{D} t} f_{\mathrm{LM}}-\frac{f_{\mathrm{LM}}}{g^{2}} \frac{\mathcal{D}}{\mathcal{D} t} g \\
& =\left.p_{r}(t) \frac{1}{f_{\mathrm{LM}}} \frac{D^{\prime}}{D t} f_{\mathrm{LM}}\right|_{\left[z_{r}(t), t\right]},
\end{aligned}
$$

having again made use of Eq. (64), the definition of the operator $\mathcal{D} / \mathcal{D} t$, as well as $C_{\mathrm{TP}}\left(f_{\mathrm{LM}}\right)=0$. One notices that collisions have no effect on the $p$-weights for the considered local Maxwellian background $f_{\mathrm{LM}}$. In the neoclassical limit, there is, in fact, no collisionless dynamics for the $p$-weights as $D f_{\mathrm{LM}} / D t_{\text {neo }}=0$. Note that in the absence of collisions, the two weights are closely coupled in the global case $\left(D^{\prime} / D t\right.$ $=D / D t)$ by the relation $\mathrm{d} / \mathrm{d} t\left(p_{r}+w_{r}\right)=0$, which, in fact, reflects the invariance of both the full distribution $f$ and the marker distribution $g$ along the collisionless trajectories. This relation also enables to express the $p$-weight in terms of the $w$-weight, thus illustrating how a single weight is sufficient for carrying out collisionless simulations.

\section{B. Interpretation of marker weights}

Dropping the terms in the operator $\delta / \delta t$ going from Eq. (68) to Eq. (70) leads to the set of weight equations (72) and (73), which are, in fact, exact when correctly reinterpreted. This was shown and discussed in detail in Refs. 11 and 13. What needs to be reconsidered, however, are the original definitions of the weights $w_{r}(t)$ and $p_{r}(t)$ in terms of the weight fields $W(z, t)$ and $P(z, t)$ given by Eqs. (60) and (63), respectively, with significant practical consequences. As a result of the modifications leading to Eq. (70), once the system has evolved from its initial state and undergone collisional processes, two markers can meet at the same phasespace point with different weights. In other words, a spreading of weights occurs over time at each phase-space point $z$ and at a rate that is proportional to the collision frequency. The original definition of the weights (60) and (63) as the (single) value of the weight fields at the marker positions, used to initialize the weights at $t=0$, is thus clearly violated at any time other than $t=0$. It is, in fact, only the statistical average over all weights of markers in the vicinity of a phase-space point, which provides an estimate of the weight fields $W$ and $P$, not the weights of a single marker. Contrary to a collisionless system, the weights $w$ and $p$ must thus be considered as additional effective dimensions of the numerical system, whose extensions grow on the collisional time scale. The practical price to pay for this weight spreading is increasing numerical noise as the simulation evolves.

\section{Discretization of the Lorentz e-i collision operator}

The electron-ion collision operator defined by Eq. (19) contributes to the test particle operator $C_{\mathrm{TP}}$, which, through random kicks, affects the marker trajectories in velocity space. Effectively, only the pitch angle direction $\xi$ of the markers is modified. No background reaction operator $C_{\mathrm{BR}}$ appears in the electron-ion collisions, and the $w$-weight equation is thus unaffected by this type of collisions. Rewriting the $e-i$ collision operator (21) as follows:

$$
C_{e i}[f]=-\nu_{e i}(v)\left\{\frac{\partial}{\partial \xi}(2 \xi f)+\frac{\partial^{2}}{\partial \xi^{2}}\left[\left(1-\xi^{2}\right) f\right]\right\},
$$

enables to conveniently identify the corresponding drag and diffusion in the $\xi$-direction. The random increment $\Delta \xi$ on the pitch angle variable resulting from $e-i$ collision over an infinitesimally small time step $\Delta t$ may thus be evaluated as

$$
\Delta \xi=-2 \xi \nu_{e i}(v) \Delta t+R \sqrt{2\left(1-\xi^{2}\right) \nu_{e i}(v) \Delta t},
$$

where $R$ is a random number sampled from a PDF with average 0 and variance 1 . In practice, making use of Eq. (75) for a finite time step may, however, result in $\xi_{\text {out }}=\xi+\Delta \xi$ falling outside of its correct interval $[-1,1]$. For the purpose of conveniently treating $e-i$ collisions, one thus temporarily expands the two-dimensional gyrokinetic velocity space back to three dimensions as follows: at any configuration space point $\vec{R}$, one starts by defining $\hat{e}_{\perp}$ as an arbitrary unitary vector perpendicular to $\hat{b}$. The incoming velocity, i.e., before the collisional kick, of a given particle at $\vec{R}$ is defined as $\vec{v}_{\text {in }}=v_{\|} \hat{b}+v_{\perp} \hat{e}_{\perp}$, with $v_{\perp}=\sqrt{2 B(\vec{R}) \mu / m}$. As shown in Fig. 1 , one then expands the velocity space to three dimensions by introducing a first orthonormal coordinate system $(x, y, z)$ with $\hat{e}_{z}:=\hat{b}$ and $\hat{e}_{y}:=\hat{e}_{\perp}$. A second orthonormal coordinate system $\left(x^{\prime}, y^{\prime}, z^{\prime}\right)$ is then defined such that $\hat{e}_{x^{\prime}}:=\hat{e}_{x}$ and $\hat{e}_{z^{\prime}}:=\vec{v}_{i n} / v_{i n}$. Let us introduce the spherical angles $(\theta, \varphi)$ associated with the system $\left(x^{\prime}, y^{\prime}, z^{\prime}\right)$. In these variables, the $e-i$ collision operator reads as 


$$
C_{e i} f=-\nu_{e i}(v)\left[\frac{1}{\sin \theta} \frac{\partial}{\partial \theta}\left(\sin \theta \frac{\partial f}{\partial \theta}\right)+\frac{1}{\sin ^{2} \theta} \frac{\partial^{2} f}{\partial \varphi^{2}}\right] .
$$

One clearly has $\theta_{\text {in }}=0$. The angles $\theta_{\text {out }}=\Delta \theta$ and $\varphi_{\text {out }}$ for the outgoing velocity following the kick are obtained by taking two random angles: $\varphi_{\text {out }}$ from a uniform distribution between 0 and $2 \pi$, while $\Delta \theta=2 R \sqrt{\nu_{e i}(v) \Delta t}$ is computed making use of $R$ sampled from a PDF of average 0 and variance 1 . The new velocity reads as

$$
\begin{aligned}
\vec{v}_{\text {out }}= & v_{\text {in }}\left[\sin (\Delta \theta) \cos (\varphi) \hat{e}_{x^{\prime}}+\sin (\Delta \theta) \sin (\varphi) \hat{e}_{y^{\prime}}\right. \\
& \left.+\cos (\Delta \theta) \hat{e}_{z^{\prime}}\right] .
\end{aligned}
$$

Note that the property $v_{\text {in }}^{2}=v_{\text {out }}^{2}$ is verified exactly, i.e., with no discretization error. Performing the reverse change of coordinates from $\left(x^{\prime}, y^{\prime}, z^{\prime}\right)$ to $(x, y, z)$ finally gives

$$
\begin{aligned}
v_{\|, \text {out }} & =\vec{v}_{\text {out }} \cdot \hat{e}_{z}=\vec{v}_{\text {out }} \cdot\left(-\sqrt{1-\xi_{\text {in }}^{2}} \hat{e}_{y^{\prime}}+\xi_{\text {in }} \hat{e}_{z^{\prime}}\right) \\
& =v_{\text {in }}\left[-\sin (\Delta \theta) \sin (\varphi) \sqrt{1-\xi_{\text {in }}^{2}}+\xi_{\text {in }} \cos (\Delta \theta)\right], \\
v_{\perp, \text { out }}^{2} & =v_{\text {in }}^{2}-v_{\|, \text {out }}^{2},
\end{aligned}
$$

providing the outgoing gyrokinetic variables $\left(v_{\|, \text {out }}, \mu_{\text {out }}\right.$ $\left.=m v_{\perp, \text { out }}^{2} / 2 B\right)$. The outgoing pitch angle variable $\xi_{\text {out }}=-\sin (\Delta \theta) \sin (\varphi) \sqrt{1-\xi_{\text {in }}^{2}}+\xi_{\text {in }} \cos (\Delta \theta)$ resulting from Eq. (78) verifies $\xi_{\text {out }} \in[-1,1]$ by construction even for finite values of $\Delta \theta$, which thus resolves the practical issue related to the scheme based on Eq. (75).

\section{Discretization of the self-collision operator}

\section{Handling the test particle operator $C\left[f_{\mathrm{LM}}, \delta f_{\mathrm{LM}}\right]$}

Similar to the Lorentz operator, this part of the linearized self-collision operator is accounted for through appropriately defined random kicks to marker trajectories. Introducing again $(x, y, z)$ and $\left(x^{\prime}, y^{\prime}, z^{\prime}\right)$, the orthogonal systems defined in Sec. III C, and making use of the Rosenbluth potentials defined by Eqs. (28) and (29), the velocity increments related to the Langevin method for like-species collisions can be written, according to Eqs. (25) and (26), as ${ }^{13}$

$$
\begin{aligned}
& \Delta v_{x^{\prime}}=v_{\text {th }} \sqrt{\frac{K(\mathrm{v}) \bar{\nu} \Delta t}{2}} R_{1}, \\
& \Delta v_{y^{\prime}}=v_{\text {th }} \sqrt{\frac{K(\mathrm{v}) \bar{\nu} \Delta t}{2}} R_{2}, \\
& \Delta v_{z^{\prime}}=-H(\mathrm{v}) v \bar{\nu} \Delta t+v_{\text {th }} \sqrt{H(\mathrm{v}) \bar{\nu} \Delta t} R_{3},
\end{aligned}
$$

where $R_{1}, R_{2}$, and $R_{3}$ are three independent random numbers sampled from a PDF with average 0 and variance 1 . Going back to gyrokinetic velocity variables provides $\left(v_{\|, \text {out }}, \mu_{\text {out }}=m v_{\perp, \text { out }}^{2} / 2 B\right)$ with

$$
v_{\|, \mathrm{out}}=\frac{1}{v_{\text {in }}}\left[-\Delta v_{y^{\prime}} v_{\perp, \text { in }}+\left(v_{\text {in }}+\Delta v_{z^{\prime}}\right) v_{\|, \text {in }}\right],
$$

$$
v_{\perp, \text { out }}^{2}=\Delta v_{x^{\prime}}^{2}+\frac{1}{v_{\text {in }}^{2}}\left[\Delta v_{y^{\prime}} v_{\|, \text {in }}+\left(v_{\text {in }}+\Delta v_{z^{\prime}}\right) v_{\perp, \text { in }}\right]^{2} .
$$

Notice that by working temporarily in the expanded threedimensional velocity space avoids similar practical problems related to the bounded pitch angle variable space as for the $e-i$ Lorentz collision operator.

\section{Handling the background reaction operator $C\left[\delta f_{\mathrm{LM}}, \boldsymbol{f}_{\mathrm{LM}}\right]$}

The background reaction operator $C\left[\delta f_{\mathrm{LM}}, f_{\mathrm{LM}}\right]$ is handled in the $w$-weight equation according to Eq. (72). As collisions are stepped separately from the collisionless dynamics by making use of a time splitting approach, only the collisional part of the weight evolution (72) is addressed here,

$$
\frac{\mathrm{d} w_{r}}{\mathrm{~d} t}=-\left.p_{r} \frac{1}{f_{\mathrm{LM}}} C\left[\delta f_{\mathrm{LM}}, f_{\mathrm{LM}}\right]\right|_{\left(z_{r}(t), t\right)},
$$

the term $D f_{\mathrm{LM}} / D t$ having been dropped as it relates to collisionless dynamics. The functional form for $C\left[\delta f_{\mathrm{LM}}, f_{\mathrm{LM}}\right]$ is chosen as in Eq. (32). A straightforward estimation for the fields $\delta \mathcal{P}_{\|}$and $\delta \mathcal{E}$ would be given by ${ }^{10,12}$

$$
\begin{aligned}
& \delta \mathcal{P}_{\|}(\vec{x})=\left.\sum_{r} w_{r} \frac{\delta v_{\|, r}}{\delta t}\right|_{\text {coll }} \delta\left(\vec{x}-\vec{x}_{r}\right), \\
& \delta \mathcal{E}(\vec{x})=\left.\sum_{r} w_{r} \frac{\delta\left(v_{r}^{2}\right)}{\delta t}\right|_{\text {coll }} \delta\left(\vec{x}-\vec{x}_{r}\right),
\end{aligned}
$$

where $\delta /\left.\delta t\right|_{\text {coll }}$ stands for the variations due to self-collisions on the background. However, such an approach does not ensure exact conservation of mass, parallel momentum, and energy as it suffers from numerical errors related to the finite time stepping and to the statistical description with a finite number of markers. A modified scheme suggested by Satake et al. $^{32}$ has been implemented in ORB5, which ensures conservation of the three velocity moments within each configuration space bin $\alpha$ exactly, i.e., to numerical round-off. Let us briefly rederive here the underlying algorithm. The integration of Eq. (83) follows the test particle scattering of markers off the background, which, as described in Sec. III D 1, accounts for the contribution $C\left[f_{\mathrm{LM}}, \delta f_{\mathrm{LM}}\right]$ of the linearized self-collision operator. As a result of this scattering, each marker $r$, having an incoming velocity $\vec{v}_{\mathrm{in}, r}$, is reassigned a new outgoing velocity $\vec{v}_{\text {out }, r}=\vec{v}_{\text {in }, r}+\Delta \vec{v}$. The integration of Eq. (83) can be written similarly as $w_{\text {out }, r}=w_{\text {in }, r}+\Delta w_{r}$. To compute $\Delta w_{r}$ according to Eq. (83), one considers the following functional form:

$$
\begin{aligned}
\Delta w_{r}= & -\frac{p_{r}}{n_{\alpha}}\left[(1-3 \sqrt{\pi} G(\mathrm{v})) \Delta N_{\alpha}+6 \sqrt{\pi} H(\mathrm{v}) \frac{\Delta P_{\| \alpha} v_{\| \text {out }, r}}{v_{\text {th } \alpha}^{2}}\right. \\
& \left.+\sqrt{\pi} G(\mathrm{v}) \frac{\Delta E_{\alpha}}{v_{\text {th } \alpha}^{2}}\right],
\end{aligned}
$$

where the functions $H$ and $G$ given by Eqs. (28) and (30) are evaluated at the normalized outgoing velocity $\mathbf{v}=v_{\text {out }, r} / v_{\text {th }} \alpha$, with $n_{\alpha}$ and $v_{\text {th } \alpha}$ being the background density and thermal 
velocity at the center of the bin $\alpha$ in which the marker is currently localized. The parameters $\Delta N_{\alpha}, \Delta P_{\| \alpha}$, and $\Delta E_{\alpha}$, specific to each bin, are to be determined so as to ensure exact conservation of the velocity moments. In particular, note the additional term proportional to $\Delta N_{\alpha}$ in Eq. (86) compared to Eq. (32), whose purpose is to ensure conservation of mass to round-off despite numerical discretization errors. Conservation of moments within the bin $\alpha$ of volume $\Delta V_{\alpha}$ over the full discretized collision step reads as

$$
\begin{gathered}
\sum_{r \in \alpha} w_{\mathrm{in}, r}=\sum_{r \in \alpha} w_{\mathrm{out}, r} \Rightarrow \frac{1}{\Delta V_{\alpha}} \sum_{r \in \alpha} \Delta w_{r}=0, \\
\sum_{r \in \alpha} w_{\mathrm{in}, r} v_{\| \mathrm{in}, r}=\sum_{r \in \alpha} w_{\mathrm{out}, r} v_{\| \text {out }, r} \\
\Rightarrow \frac{1}{\Delta V_{\alpha}} \sum_{r \in \alpha} \Delta w_{r} v_{\| \text {out }, r}=-\Delta \mathcal{P}_{\| \alpha}, \\
\sum_{r \in \alpha} w_{\mathrm{in}, r} v_{\mathrm{in}, r}^{2}=\sum_{r \in \alpha} w_{\mathrm{out}, r} v_{\mathrm{out}, r}^{2} \\
\Rightarrow \frac{1}{\Delta V_{\alpha}} \sum_{r \in \alpha} \Delta w_{r} v_{\mathrm{out}, r}^{2}=-\Delta \mathcal{E}_{\| \alpha},
\end{gathered}
$$

where $\Delta \mathcal{P}_{\| \alpha}=\sum_{r} w_{\mathrm{in}, r} \Delta v_{\|, r} / \Delta V_{\alpha}$ and $\Delta \mathcal{E}_{\| \alpha}=\sum_{r} w_{\mathrm{in}, r} \Delta\left(v_{r}^{2}\right) / \Delta V_{\alpha}$, having defined $\Delta v_{\|, r}=v_{\| \text {out }, r}-v_{\| \text {in }, r}$ and $\Delta\left(v_{r}^{2}\right)=v_{\text {out }, r}^{2}-v_{\mathrm{in}, r}^{2}$ Inserting Eq. (86) into Eqs. (87)-(89) provides a linear system of three equations for the parameters $\left(\Delta N_{\alpha}, \Delta P_{\alpha}, \Delta E_{\alpha}\right)$, which can be written as follows:

$$
\mathbf{M}_{\alpha}\left(\begin{array}{c}
\Delta N_{\alpha} \\
\Delta P_{\alpha} \\
\Delta E_{\alpha}
\end{array}\right)=\left(\begin{array}{c}
0 \\
\Delta \mathcal{P}_{\| \alpha} \\
\Delta \mathcal{E}_{\alpha}
\end{array}\right),
$$

with

$$
\begin{aligned}
\mathbf{M}_{\alpha}= & \frac{1}{\Delta V_{\alpha}} \sum_{r \in \alpha} \frac{p_{r}}{n_{\alpha}}\left[\begin{array}{c}
1 \\
v_{\| \text {out }, r} \\
v_{\text {out }, r}^{2}
\end{array}\right] \\
& \times\left([1-3 \sqrt{\pi} G], 6 \sqrt{\pi} H \frac{v_{\| \text {out }, r}}{v_{\text {th } \alpha}^{2}}, \sqrt{\pi} G \frac{1}{v_{\text {th } \alpha}^{2}}\right) .
\end{aligned}
$$

The matrix $\mathbf{M}_{\alpha}$ and the RHS of system (90) must be computed separately for each bin $\alpha$ and at each collisional time step. The solution of Eq. (90) provides the coefficients $\left(\Delta N_{\alpha}, \Delta P_{\alpha}, \Delta E_{\alpha}\right)$, which are then inserted into Eq. (86), thus enabling to finally compute the weight increments $\Delta w_{r}$ and fully complete the self-collision stepping. The algorithm clearly ensures exact numerical conservation of the velocity moments by construction. In the limit of large number of markers per bin, as well as $\Delta V_{\alpha} \rightarrow 0$, the scheme obviously converges to the original equation (32) for the background reaction. In particular, note that in this limit, the matrix $\mathbf{M}_{\alpha}$ reduces to
TABLE I. Run parameters for local neoclassical electron transport.

$$
\begin{aligned}
& \text { Profile } 1 \quad a / R_{0}=0.36 \quad 1 / \rho^{*}=140 \quad \Delta_{T}=0.3 \quad \Delta_{n}=0.3 \\
& \mathbf{M}_{\alpha}=\int \mathrm{d}^{3} v \frac{f_{\mathrm{LM}}}{n_{0}}\left(\begin{array}{c}
1 \\
v_{\|} \\
v^{2}
\end{array}\right)\left([1-3 \sqrt{\pi} G], 6 \sqrt{\pi} H \frac{v_{\|}}{v_{\mathrm{th}}^{2}}, \sqrt{\pi} G \frac{1}{v_{\mathrm{th}}^{2}}\right) \\
& \text { = I }
\end{aligned}
$$

so that, according to Eq. (90), $\Delta N_{\alpha}=0, \Delta P_{\alpha}=\Delta \mathcal{P}_{\| \alpha}$, and $\Delta E_{\alpha}=\Delta \mathcal{E}_{\alpha}$ as in Eq. (32).

\section{NEOCLASSICAL TRANSPORT SIMULATIONS}

Neoclassical transport benchmarks, both for ions and electrons, are shown in order to validate the implementation of the collision operators described in Sec. III. Simulations are initialized here with $\delta f_{\mathrm{LM}}(t=0)=0$. Invoking the axisymmetry of the neoclassical equilibrium which may be assumed in a tokamak, the binning for the background reaction operator described in Sec. III D 2 needs only to be performed in the poloidal plane. We use typically 64 bins in the radial direction and 128 bins in the poloidal direction. The typical number of markers used in the neoclassical simulations is $100 \times 10^{6}$ for the global case with self-consistent electric field and $20 \times 10^{6}$ for the local case assuming no electric field. The time step and the total simulation time are dictated by the transit time and the collision time, respectively, for the banana regime and inversely for the collisional regime.

\section{A. Benchmarks for local neoclassical electron transport: No electric field}

The results of this subsection, obtained with the parameters given in Table I, benchmark electron neoclassical transport computed with ORB5 against previous numerical and analytical results. One considers the local neoclassical limit case, i.e., solving Eq. (8) for the electron distribution, and no electric field. The ion dynamics is not taken into account here, as it is anyway decoupled from the electron dynamics in this local limit. Both the full linearized electron collision operator, i.e., considering both $e-e$ self-collisions and $e-i$ pitch angle scattering, as well as the simplified Lorentz model, i.e., considering $e-i$ collisions alone, are used. Note that the latter is equivalent to the former when setting the ionization degree $Z \rightarrow \infty$. The particle flux $\Gamma$ produced by a density gradient alone, considering $\kappa_{n}=5$ and $\kappa_{T}=0$, is shown in Fig. 2, and the energy flux $Q_{\text {kin }}$ produced by a temperature gradient alone, considering $\kappa_{n}=0$ and $\kappa_{T}=5$, is shown in Fig. 3. These results are benchmarked against analytical fits to results from the Eulerian-Fokker-Planck code CQL3D (Ref. 17) and analytical results derived for the Lorentz model $(Z \rightarrow \infty)$ in the review by Hinton and Hazeltine. ${ }^{31}$ Figures 2(a) and 3(a) present the fluxes at the radial position $s=0.425$ as a function of the effective collisionality $\nu^{*}$, ranging from the low collisionality (banana) regime for $\nu^{*} \ll 1$ up to the collisional regime for $\nu^{*} \gg 1$. Figures 2(b) and 3(b) present the neoclassical fluxes as a function of radius, ac- 

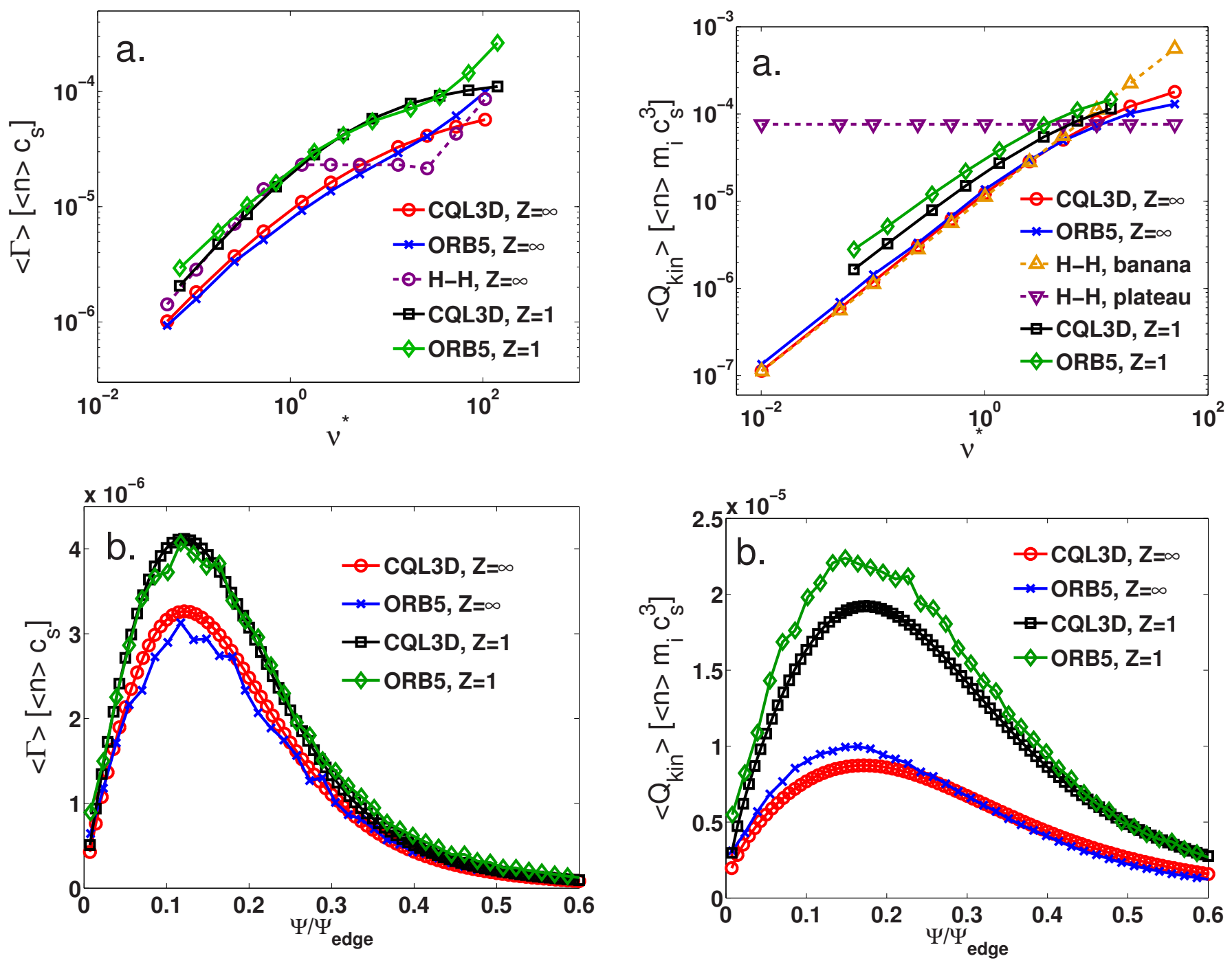

FIG. 2. (Color online) Neoclassical electron particle flux $\Gamma$ for $\kappa_{n}=5, \kappa_{T}$ $=0$, as a function of (a) the effective collisionality $\nu^{*}$ at the given radial position $s=0.425$ and (b) as a function of radius with a collisionality accounting for the density and temperature profiles, $\nu^{*}\left(s=s_{0}\right)=0.18$. ORB5 results are shown for $Z=\infty$ and $Z=1$ and compared with analytical fits to CQL3D results for $Z=\infty$ and $Z=1$, as well as analytical results for the Lorentz model given in the review by Hazeltine and Hinton.

counting for the radial variations of density and temperature profiles both for computing the local gradients as well as for estimating collisionality. The collisionality at $s=s_{0}=0.5$ was fixed to $\nu^{*}=0.18$ and $\nu^{*}=0.68$ for Figs. 2(b) and 3(b), respectively. The agreement between ORB5, CQL3D, and the HintonHazeltine results may be considered satisfactory, in particular, considering that the CQL3D curves are only obtained through approximate fits to the actual simulation results and the Hinton-Hazeltine are the result of approximate analytical derivations.

\section{B. Neoclassical ion transport with self-consistent electric field}

The neoclassical theory predicts the establishment of a radial electric field leading to an axisymmetric equilibrium, which was first computed in a global simulation by Wang et al. using the FORTEC code. ${ }^{20}$ In order to study a neoclas-

FIG. 3. (Color online) Neoclassical electron energy flux $Q_{\text {kin }}$ for $\kappa_{n}$ $=0, \kappa_{T}=5$, as a function of (a) the effective collisionality $\nu^{*}$ at the given radial position $s=0.425$ and (b) as a function of radius with a collisionality accounting for the density and temperature profiles, $\nu^{*}\left(s=s_{0}\right)=0.68$. ORB5 results are shown for $Z=\infty$ and $Z=1$ and compared with analytical fits to CQL3D results for $Z=\infty$ and $Z=1$, as well as analytical results for the Lorentz model given in the review by Hazeltine and Hinton.

sical equilibrium with self-consistent electric field, we solve the global drift-kinetic equation for ions. Indeed, as neoclassical simulations involve electric fields with long wavelengths, it is relevant to neglect FLR effects even in the collisionless dynamics. Equation (7) is solved, together with the quasineutrality equation (15) or Eq. (16), assuming axisymmetry, i.e., keeping only the toroidal Fourier mode $n=0$ of the fluctuating fields $\delta f_{\mathrm{LM}}$ and $\phi$. Both adiabatic and kinetic electron dynamics will be considered in the following. The standard neoclassical approximation retains only the flux surface averaged potential and thus neglects the poloidal components of the field. The specific parameters of the simulations are given in Tables II and III (cases 1 and 2, respectively). If not mentioned otherwise, a standard run is carried out with full marker motion, i.e., in the global case, and a purely radial electric field. The radial electric field $E_{r}$ is obtained from the flux surface average $\langle\phi\rangle$ of the electro- 
TABLE II. Run parameters, ion transport benchmark, case 1 .

\begin{tabular}{cccc}
\hline \hline Profile 2 & $a / R_{0}=0.36$ & $T_{e} / T_{i}=1$ & $\nu_{i}^{*}(r / a=0.5)=0.03$ \\
$\kappa_{T}=3.58$ & $\kappa_{n}=0.79$ & $1 / \rho^{*}=150$ & $\Delta_{T_{i}}=\Delta_{n}=0.3$ \\
\hline
\end{tabular}

static potential $\phi$, solution to the quasineutrality equation: $E_{r}=-\partial\langle\phi\rangle / \partial r$. The considered ion species is deuterium. The electron temperature is the same as the ion temperature. In global simulations, collision frequencies are estimated locally, i.e., consistent with background density and temperature variations.

\section{Zonal flow damping: Rosenbluth-Hinton test}

The study of the self-consistent axisymmetric electrostatic fields in the frame of neoclassical transport is closely linked to the study of zonal poloidal flows, which play a crucial role in regulating microturbulence, ${ }^{6}$ and geodesic acoustic mode (GAM) oscillations. ${ }^{33}$ Thus, we start by presenting here linear simulations [i.e., dropping the term $\vec{v}_{E \times B} \cdot \partial \delta f_{\mathrm{LM}} / \partial \vec{R}$ of Eq. (7)] of zonal flow damping, corresponding to the so-called Rosenbluth-Hinton test. The electrons are considered adiabatic and the full axisymmetric potential $\phi_{n=0}$ is retained. The collisionless theory of zonal flow damping predicts an undamped residual value. ${ }^{34}$ Collisions are expected to lead to a decaying residual zonal flow in a characteristic time of the order of the collision time. Figure 4 shows the damping of the radial electric field $E_{r}$ at the magnetic surface $s=0.55$, for the geometrical parameters of case 2 but having set gradients to zero $\left(\kappa_{n}=\kappa_{T}=0\right)$, as usually done for carrying out the Rosenbluth-Hinton test. Oscillations, whose frequency is estimated by $\omega_{G}=\left(v_{\text {th } i} / R_{0}\right) \sqrt{7 / 2+23 /\left(7 q_{s}^{2}\right)}$ according to Ref. 35 in the drift-kinetic limit, are due to the initial excitation of GAMs, which are Landau damped. Neglecting the finiteorbit-width effects, an estimation of the damping rate is given by $\gamma_{G}=\left(v_{\text {th } i} / R_{0}\right) \exp \left(-q_{s}^{2}-0.5\right) \sqrt{2+1 / q_{s}^{2}}$. ${ }^{36}$ Defining $\varpi=1+1.6 q_{s}^{2} / \sqrt{\epsilon}$, the time evolution of the electric field may be written as follows: ${ }^{37}$

$$
\begin{aligned}
\frac{E_{r}(t)}{E_{r}(0)}= & \left(1-\frac{1}{\varpi}\right) \exp \left(-\gamma_{G} t\right) \cos \left(\omega_{G} t\right) \\
& +\frac{1}{\varpi} \exp \left(\frac{\beta^{2} t}{\varpi^{2}}\right)\left[1-\operatorname{erf}\left(\frac{\beta \sqrt{t}}{\varpi}\right)\right],
\end{aligned}
$$

where erf is the error function defined in Sec. II B. The small long-time collisional response has been neglected here. Considering a finite collisionality, we define $\beta=3 \pi q_{s}^{2} \sqrt{\bar{\nu}} /\left(\epsilon \ln ^{3 / 2}[16 \sqrt{\epsilon / \bar{\nu} t}]\right)$. In the collisionless limit, $\beta \rightarrow 0$ and Eq. (93) becomes thus ${ }^{34}$

TABLE III. Run parameters, ion transport benchmark, case 2.

\begin{tabular}{lccc}
\hline \hline Profile 2 & $a / R_{0}=0.2$ & $T_{e} / T_{i}=1$ & $0.012<\nu_{i}^{*}(r / a=0.5)<12$ \\
$\kappa_{T}=\kappa_{n}=1.2$ & $\Delta_{T_{i}}=0.2$ & $\Delta_{n}=0.2$ & $1 / \rho^{*}=150$ \\
\hline \hline
\end{tabular}

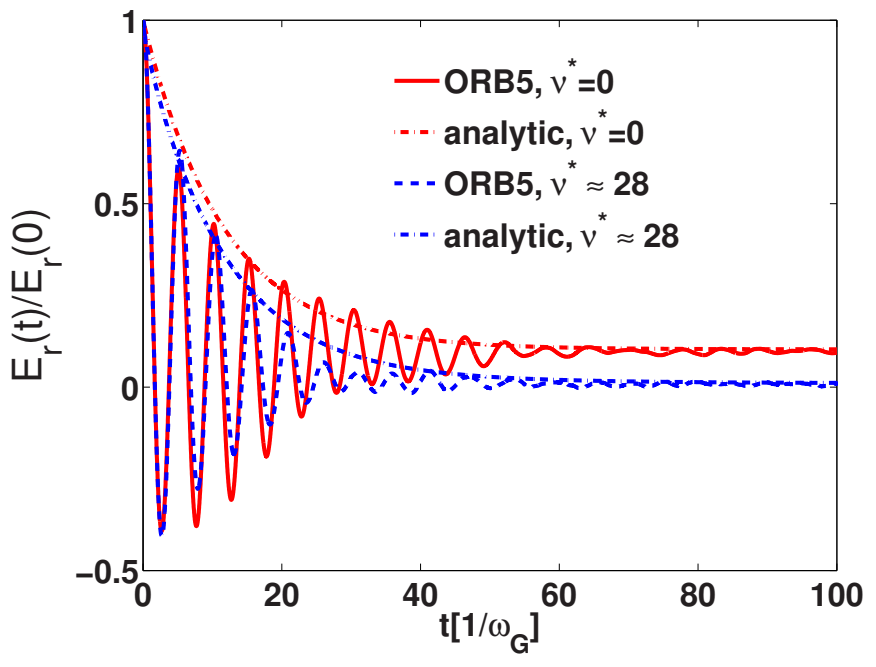

FIG. 4. (Color online) Rosenbluth-Hinton test at $s=0.55$ considering an axisymmetric field, linear dynamics, without pressure gradient. The time evolution of the radial electric field $E_{r}=-\partial\langle\phi\rangle / \partial r$ predicted by ORB5 is compared to the analytical behavior derived by Rosenbluth and Hinton for the collisionless and the collisional cases, respectively.

$$
\frac{E_{r}(t)}{E_{r}(0)}=\left(1-\frac{1}{\varpi}\right) \exp \left(-\gamma_{G} t\right) \cos \left(\omega_{G} t\right)+\frac{1}{\varpi} .
$$

The predicted damping rates and residuals, shown by dashed-dotted lines in Fig. 4, match well the time evolution of the radial electric field given by ORB5 at $s=0.55$, for a collisionless and a collisional run, respectively. This test is crucial in order to ensure a correct level of the zonal flow in turbulent simulations.

\section{Ion fluxes with adiabatic electrons}

In this section, a benchmark is performed against previous neoclassical numerical results obtained with both the Eulerian code GT5D and the PIC code FORTEC-3D, ${ }^{38}$ retaining only the flux surface averaged potential $(\phi=\langle\phi\rangle)$. These results are also compared with neoclassical analytical results. All the ORB5 results are within the typical range of the small discrepancies between GT5D and FORTEC-3D, and may thus be considered as consistent with other codes. Figure 5(a) presents the ion heat diffusivity $\chi_{H i}$ as a function of radius in units of $\chi_{\mathrm{GB}} a / L_{n}$ for case 1 parameters (Table II), which results exclusively from the $\delta f_{\mathrm{LM}} \vec{v}_{d} \cdot \vec{\nabla} \Psi$ contribution to the kinetic energy flux $Q_{\text {kin }}$ given by Eq. (46), and matches well the analytical predictions from Chang and Hinton, ${ }^{39}$ where the neoclassical assumption is valid, i.e., away from the magnetic axis. The force balance parameter $k$, introduced in Eq. (50), is shown around the midradius region for the same case 1 in Fig. 5(b) and compared with the analytical large aspect ratio predictions from Hinton and Hazeltine, ${ }^{31}$ as well as with the results from the moment equation approach derived by Hirshman and Sigmar. ${ }^{40}$

Figure 6 shows a collisionality scan for the ion heat diffusivity $\chi_{H i}$ and the neoclassical force balance parameter $k$ at $r=0.5 a$ for case 2 parameters (Table III). Predictions coming from the moment equation approach (Hirshman-Sigmar ${ }^{40}$ ) are shown in addition to the results derived by Hinton and 

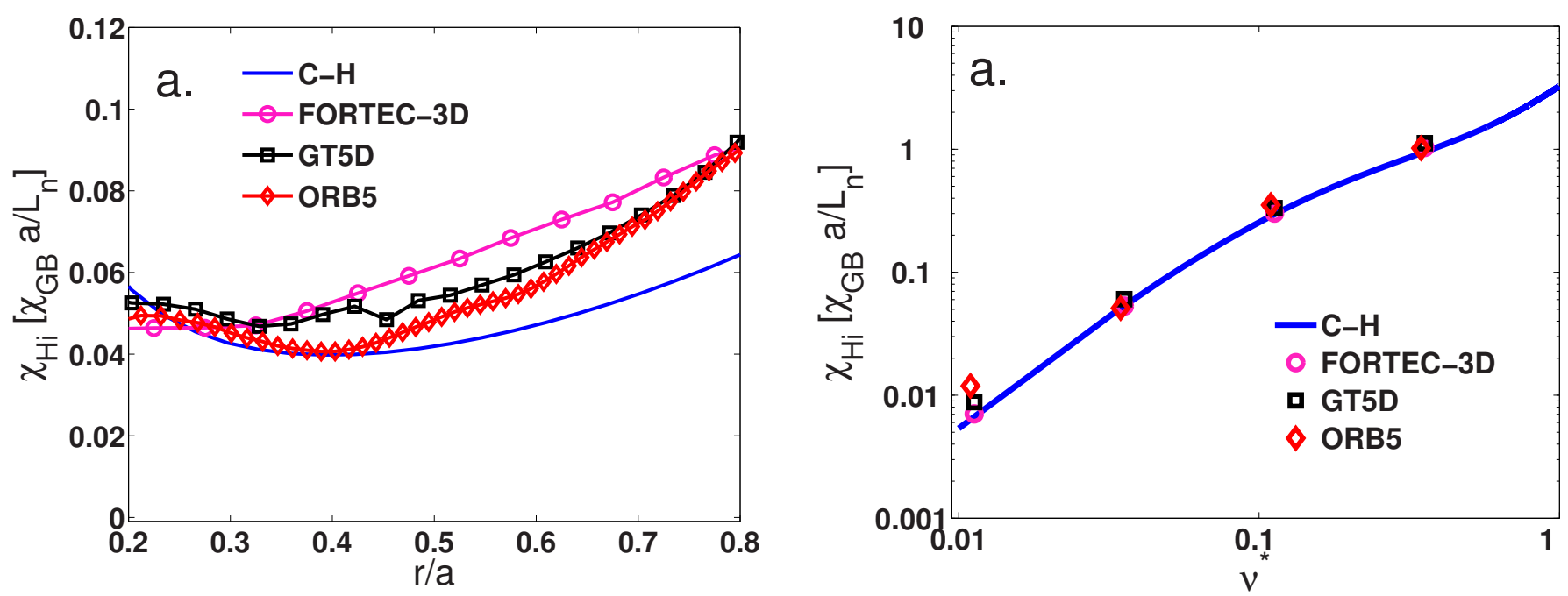

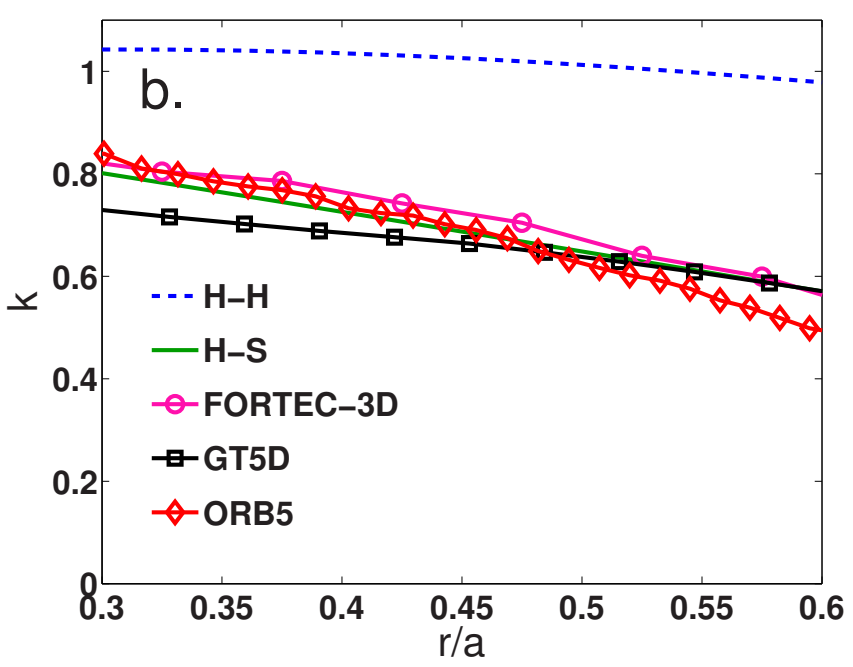

FIG. 5. (Color online) (a) Ion heat diffusivity $\chi_{H i}$ and (b) neoclassical force balance coefficient $k$ as a function of radius. Case 1 parameters, adiabatic electrons, and retaining only $\langle\phi\rangle$ from the quasineutrality equation. Global ORB5 results at time $t=\tau_{i i}(r / a=0.5)$ are compared to simulations from GT5D and FORTEC-3D, as well as analytical results from Chang and Hinton, Hinton and Hazeltine, Hirshman and Sigmar.

Hazeltine in Ref. 31 for the coefficient $k$ appearing in the force balance equation (50). In Fig. 7(a), showing the time evolution of the particle flux at $r=0.5 a$ for the same case 2 , the ambipolarity condition is verified, resulting in a vanishing ion flux for $t \rightarrow \infty$ since the electrons are assumed to respond adiabatically. The setting up of the neoclassical radial electric field at $r=0.5 a$ is shown in Fig. 7(b).

Possible effects from the poloidal Fourier modes $m \neq 0$, retained beyond the neoclassical approximation, are studied in Fig. 8, corresponding to case 1 parameters (Table II). In Fig. 8(a), the ORB5 results for $\phi=\langle\phi\rangle$ have been added for reference. Considering only the kinetic contribution to the heat flux, one could be misled into interpreting this kinetic energy flux as a contribution to the thermal transport: it is not, as pointed out in Ref. 30. Actually, the potential energy flux from the background $f_{\mathrm{LM}}$ compensates the kinetic energy flux from the background $f_{\mathrm{LM}}$, leading to the same heat diffusivity level as in the standard neoclassical approxima-

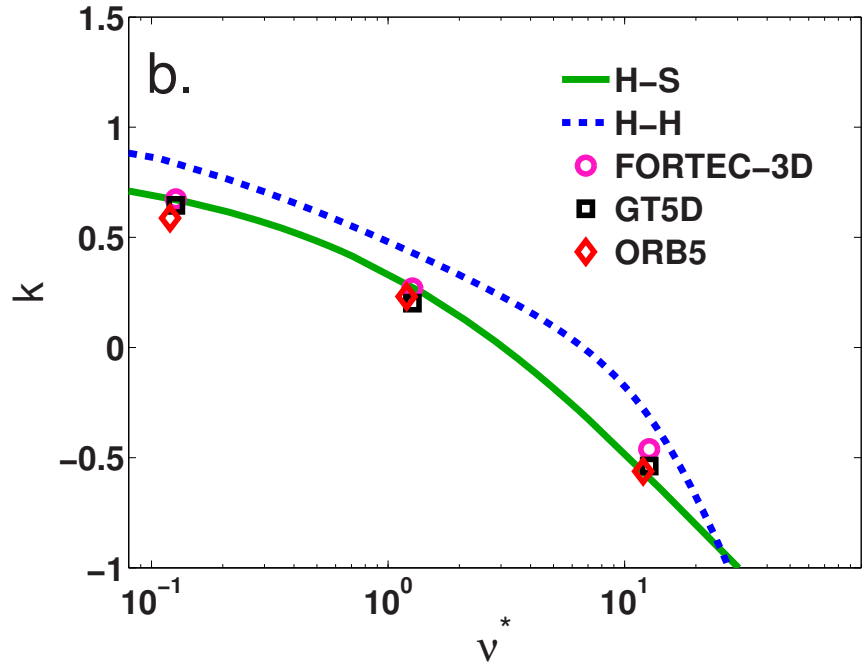

FIG. 6. (Color online) (a) Ion heat diffusivity $\chi_{H i}$ and (b) the neoclassical force balance coefficient $k$ as a function of the effective collisionality. Considering parameters of case 2 and adiabatic electrons, ORB5 results, shown for position $r / a=0.5$ and at time $t \sim \tau_{i i}(r / a=0.5)$, are compared to simulations from GT5D and FORTEC-3D, as well as analytical results from Chang and Hinton, Hinton and Hazeltine, Hirshman and Sigmar.

tion (purely radial electric field). The potential energy flux is crucial in order to treat the effects of the $m \neq 0$ poloidal modes in neoclassical transport, as discussed in Ref. 30. Retaining the $m \neq 0$ poloidal modes still ensures a total vanishing particle flux, which is shown in Fig. 8(b): the full axisymmetric potential $\phi$ leads to an inward flux related to the drift velocity $\vec{v}_{E \times B}$ acting on $f_{\mathrm{LM}}$, compensating the outward flux contribution from $\delta f_{\mathrm{LM}}$ related mainly to the magnetic drift velocity $\vec{v}_{\nabla B}+\vec{v}_{c}$. The ambipolarity is thus satisfied, as there is no electron transport, the electron response being assumed adiabatic in these simulations.

\section{Adding collisional kinetic electrons}

Case 1 simulations were repeated, considering kinetic electrons with a nonphysical ion-electron mass ratio $m_{i} / m_{e}$ $=200$ and experiencing both self-collisions as well as pitch angle scattering on ions. Thanks to the smaller mass ratio, the transit time scale separation between electrons and ions 

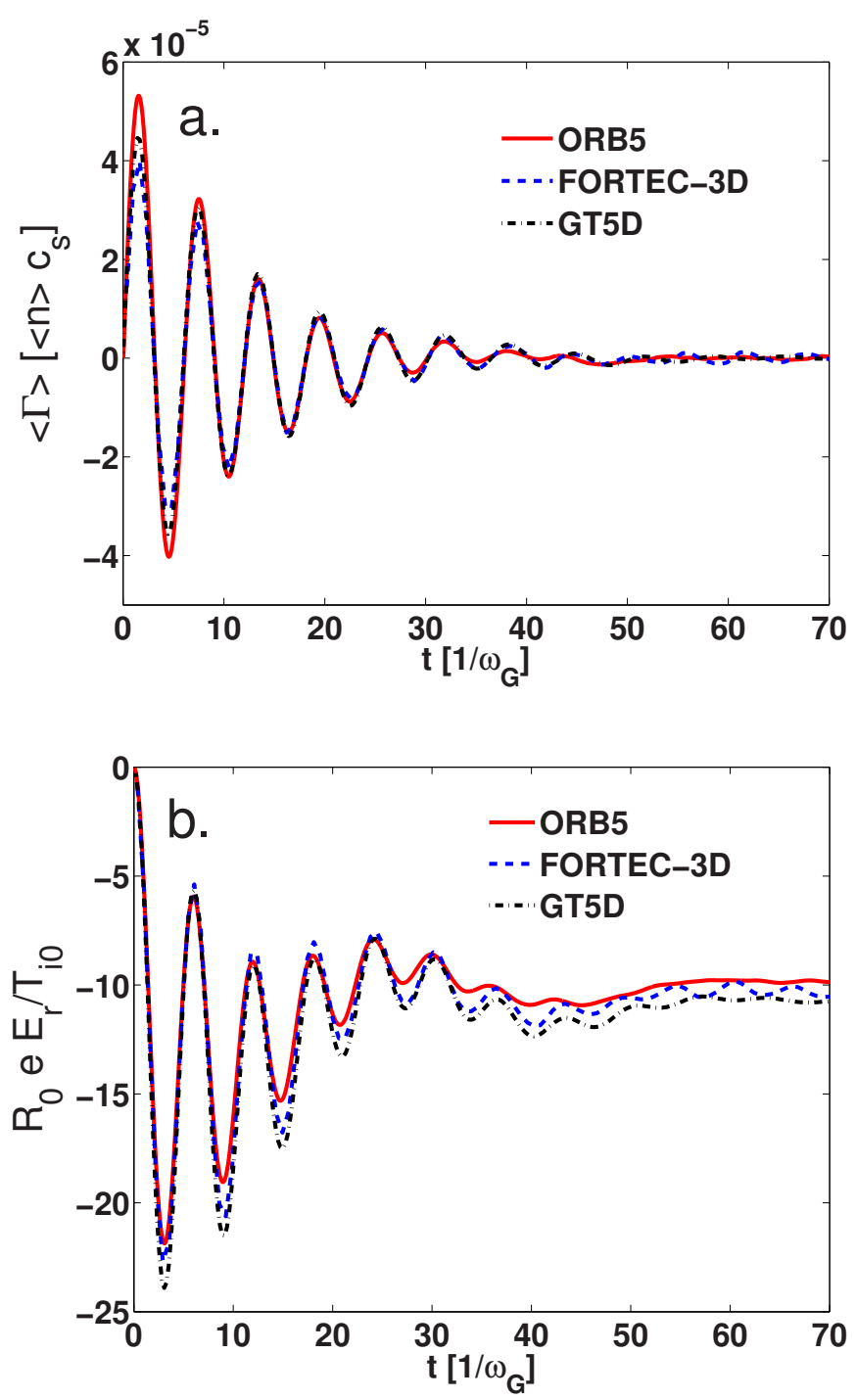

FIG. 7. (Color online) Time evolution of (a) particle flux $\Gamma$ and (b) radial electric field $E_{r}=-\partial\langle\phi\rangle / \partial r$ at $r=0.5 a$ for case 2 parameters and $\nu^{*}(r / a$ $=0.5)=0.12$. Comparison of the results from ORB5, GT5D, and FORTEC-3D. The ambipolarity is satisfied and an equilibrium neoclassical electric field is established.

$\omega_{t e} / \omega_{t i} \sim \sqrt{m_{i} / m_{e}}$ is reduced, which facilitates the multispecies kinetic simulations. The electron collisionality is artificially enhanced such that $\nu_{e e, \text { th }} / \nu_{i i, \text { th }} \sim 50$. We notice in Fig. 9 , obtained by neglecting the $(n=0, m \neq 0)$ modes, that the ratio between electron and ion heat transport is consistent with the neoclassical ordering of heat transport $\chi_{e} / \chi_{i}$ $\sim m_{e} \nu_{e e, \mathrm{th}} / m_{i} \nu_{i i, \mathrm{th}} \sim 1 / 4$. Moreover, electrons and ions both show a similar outward particle flux, which ensures the ambipolarity condition. The small discrepancy between electron and ion fluxes reflects the statistical numerical error.

The effects of kinetic electrons on the neoclassical equilibrium electric field are small, as clearly shown in Fig. 10(a). This results from the fact that the electric field ensuring the ambipolarity by shaping the particle orbits depends mainly on the ion dynamics since the average ion orbit width

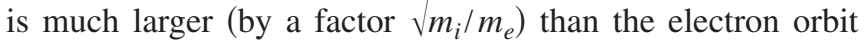
width. The orbit squeezing produced by the shear of the radial electric field is discussed in Ref. 41. Figure 10(b) shows
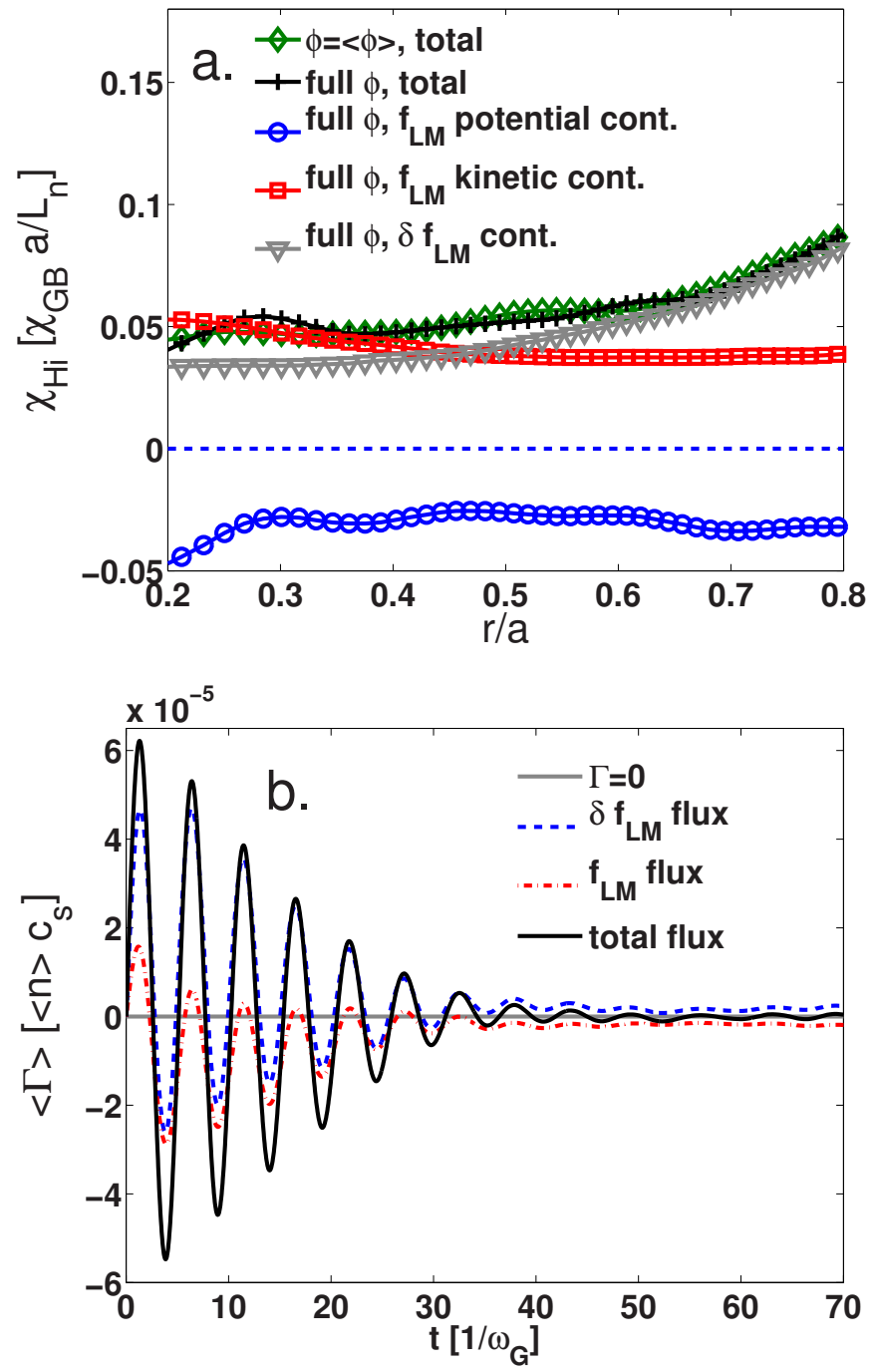

FIG. 8. (Color online) Retaining all poloidal Fourier modes of the axisymmetric potential and comparison to simulation results within the neoclassical limit of a purely radial electric field (see Fig. 5). (a) Case 1 parameters, adiabatic electrons, ion heat diffusivity at $t=0.5 \tau_{i i}(r / a=0.5)$ as a function of radius: retaining all poloidal Fourier modes does not change the heat transport level. (b) Case 2 parameters, time evolution of the particle flux at $r$ $=0.5 a$ for $\nu^{*}(r / a=0.5)=0.085$. Ambipolarity is satisfied.

that kinetic electrons lead to an important bootstrap current, mainly produced by passing electrons, while the ion contribution to this current is essentially negligible.

\section{Breakdown of the neoclassical approximation}

The neoclassical approximation is valid under the assumption that the banana width is small compared to the characteristic length of the system. In order to illustrate the limitations of this approximation, one chooses a configuration without density gradient, considering the parameters of Table IV, i.e., with relatively low value of $1 / \rho^{*}$. The computations are carried out without considering self-consistent electric fields. As discussed in Ref. 26, it is problematic to define diffusivity near the axis because of nonlocal effects and the absence of separation between the transport time scale and the neoclassical confinement time scale. However, the temperature profile does not relax significantly over our simulation times, making the study of an effective diffusivity 


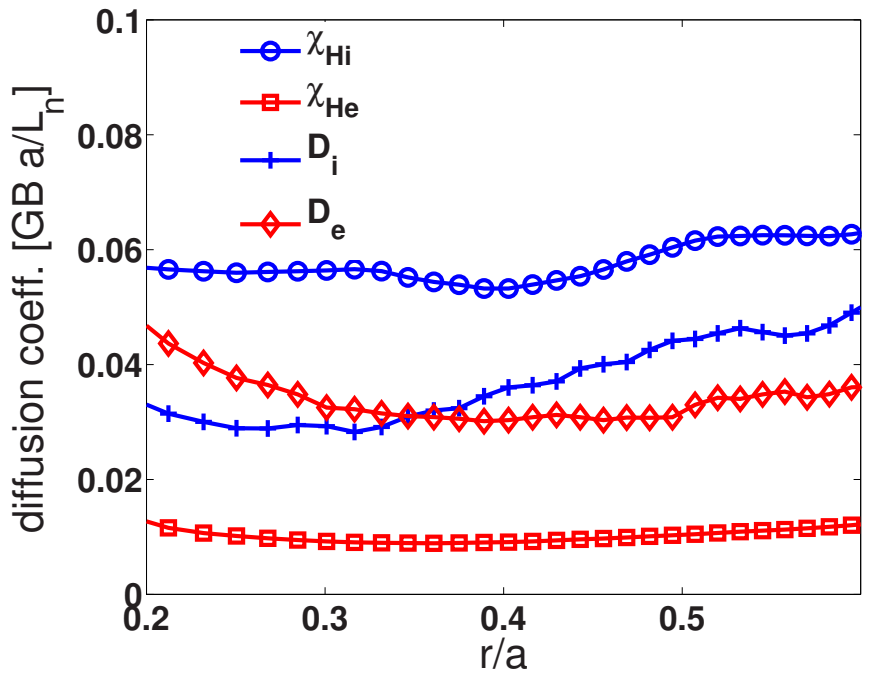

FIG. 9. (Color online) Case 1 parameters with collisional kinetic electrons. Particle and heat diffusivity of ions and electrons as a function of radius for $\phi=\langle\phi\rangle$ and time $t=2 \nu_{i i, \mathrm{th}}^{-1}=100 \nu_{e e, \mathrm{th}}^{-1}$. The results are in accordance with neoclassical transport scaling laws and ensuring ambipolarity.

relevant. Comparing global and local ORB5 simulations in Fig. 11, one notices that the neoclassical approximation is valid over the radial region $r / a \geqslant 0.45$, while it breaks down near the magnetic axis. This result is mainly explained by the fact that near the magnetic axis, trapped particles follow potato orbits whose width is of order $\lambda_{p} \sim\left(q_{s}^{2} \rho_{L}^{2} R_{0}\right)^{1 / 3}$, ${ }^{42}$ which may be significantly larger than the standard banana width $\rho_{b} \sim q_{s} \rho_{L} / \sqrt{\epsilon}$. These large orbit widths can thus become comparable to the local minor radius within a significant radial region around the magnetic axis for the large aspect ratio and large $\rho^{*}$ tokamak considered here, which violates the standard neoclassical assumption. These results are consistent with the previous ones: the reduction of the neoclassical transport near the axis with respect to the standard theory has been predicted in a low collisionality regime by a Lagrangian transport theory ${ }^{43}$ as well as by simulation results. ${ }^{42,44}$

\section{TOWARD GYROKINETIC TURBULENT SIMULATIONS}

\section{A. Noise control requirement}

Although exclusively neoclassical simulations using ORB5 have been presented here, the main purpose of the code is to carry out turbulent simulations. Due to the PIC representation considered in ORB5, a major impediment to obtaining physically relevant results is the problem of numerical noise. Noise is diagnosed in ORB5 by examining the charge density in nonresonant turbulent modes, which are very strongly Landau damped, and only arise due to sampling errors. ${ }^{45}$ In order to define a signal-to-noise ratio, the following definitions are introduced: the signal is defined by the modes of the charge density inside a certain Fourier filter $\mathcal{F}$, while the noise is defined by the modes outside the Fourier filter,
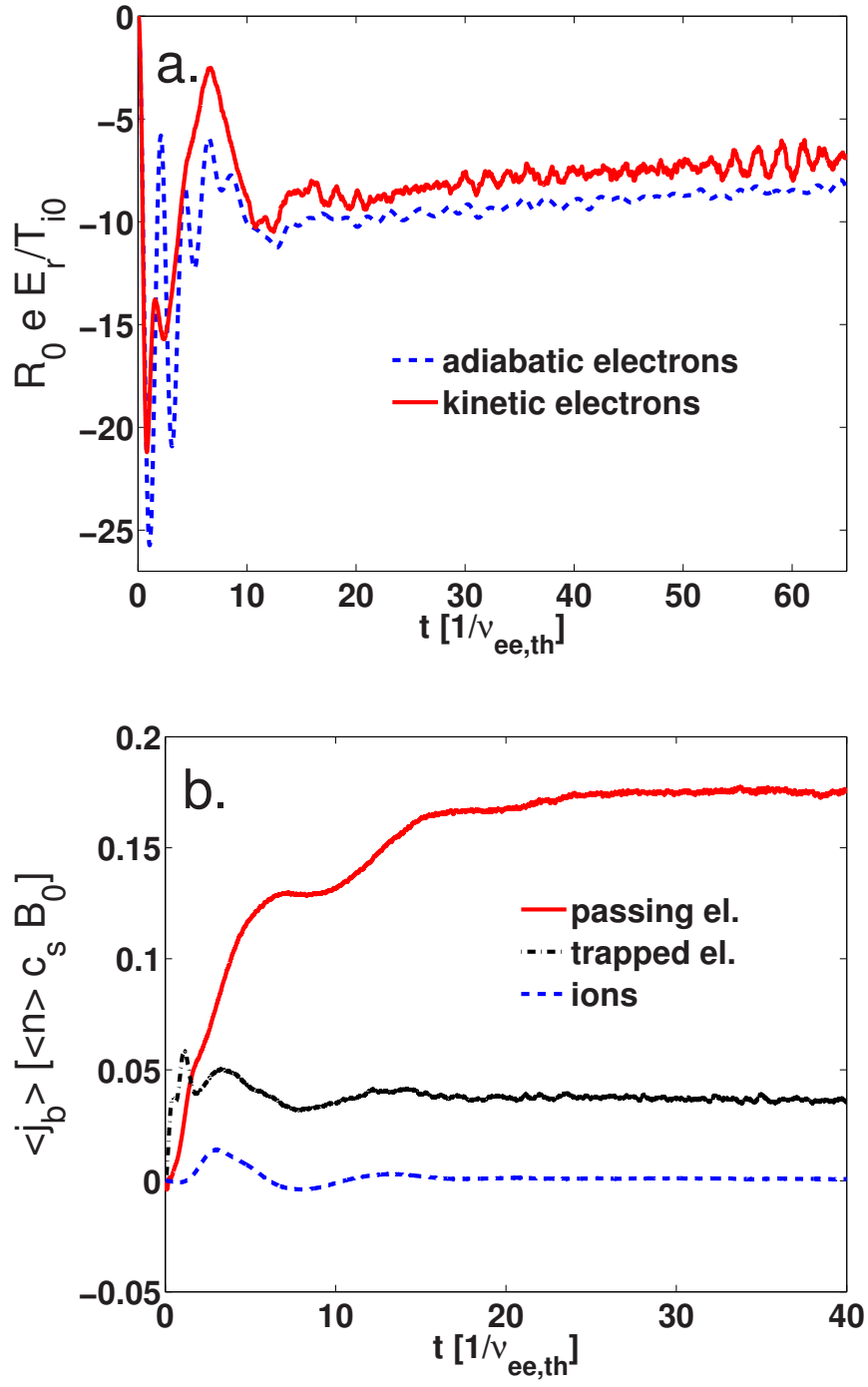

FIG. 10. (Color online) Case 1 parameters, $\phi=\langle\phi\rangle$. Kinetic electron contributions to (a) the neoclassical radial electric field at $r=0.5 a$ and (b) the bootstrap current at $r=0.5 a$. The electric field is weakly changed by considering kinetic electrons, while an important bootstrap current is produced, mainly by passing electrons.

$$
\begin{aligned}
& \text { signal }=\frac{\int_{0}^{a} d r \Sigma_{(n, m) \in \mathcal{F}}\left|\delta n^{(n, m)}(r)\right|^{2}}{a \Sigma_{(n, m) \in \mathcal{F}}}, \\
& \text { noise }=\frac{\int_{0}^{a} d r \Sigma_{(n, m) \notin \mathcal{F}}\left|\delta n^{(n, m)}(r)\right|^{2}}{a \Sigma_{(n, m) \notin \mathcal{F}}} .
\end{aligned}
$$

Invoking the fact that microturbulence is essentially aligned along the magnetic field line, the filter $\mathcal{F}$ retains Fourier modes $(n, m)$, which, at a given radial position $r$, are such that $\left|n q_{s}(r)-m\right| \leq \Delta m$, where $\Delta m=5$ is typically chosen. Let us point out that the Fourier modes are computed using a

TABLE IV. Run parameters for studying the breakdown of neoclassical approximation.

\begin{tabular}{lccc}
\hline \hline Profile 2 & $a / R=0.28$ & $T_{e} / T_{i}=1$ & $\nu_{i}^{*}(r / a=0.5)=0.1$ \\
$\kappa_{n}=0$ & $\kappa_{T}=4$ & $\Delta_{T_{i}}=0.3$ & $1 / \rho^{*}=80$ \\
\hline \hline
\end{tabular}




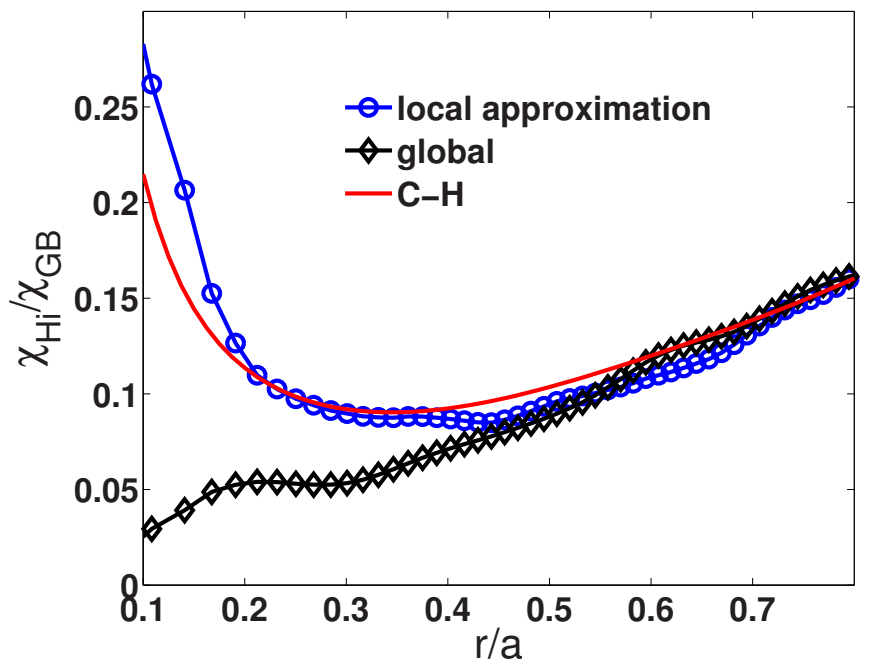

FIG. 11. (Color online) Neoclassical approximation breakdown. Reduction of the neoclassical transport predicted by ORB5 close to the magnetic axis when considering the full marker motion. In the neoclassical limit, ORB5 results match well the analytical predictions by Chang and Hinton.

straight field line poloidal angle. In the case of the axisymmetric $(n=0)$ simulations considered here, the filter $\mathcal{F}$ reduces to keeping the poloidal modes $|m| \leq \Delta m$. If the noise becomes large compared to the physical signal, the simulations become irrelevant and are not further carried out. Although noise may be kept within limits using a reasonable number of markers for carrying out neoclassical simulations, since only the axisymmetric mode $n=0$ needs to be resolved, we wish to eventually have neoclassical simulations to be followed by turbulence runs, and one must thus ensure that noise is kept as low as possible and under control both in the former and latter simulations. Noise control is especially crucial for simulations with collisions, which increase the noise due to the random kicks used in the diffusive part of the operators. Collisions indeed lead to weight spreading, adding two dimensions, $w$ and $p$, to the extended numerical phase space and thus requiring a new interpretation of the weights, ${ }^{11}$ as already discussed in Sec. III B. ORB5 was already equipped with a noise-reduction scheme, which consists of an artificial decay of the weights. ${ }^{4}$ Unfortunately, this method is not practical in a collisional simulation since the required decay rate is at least of the same order as the typical collision frequency, thus masking the physical effects of collisions. ORB5 therefore also features a newly implemented collisional noise control scheme, based on the coarse graining procedure proposed in Ref. 14. The method involves a periodic binning of markers in phase space and a reassignment of each weight to the average weight value of all markers within the bin. This approach ensures a significant weight spreading reduction. The coarse graining in ORB5 will be explained in detail in a forthcoming paper. Its beneficial effect on the noise level is nonetheless already illustrated here in the case of a neoclassical test based on the CYCLONE case, ${ }^{46}$ performed with adiabatic electrons and at low collisionality: $\nu^{*}(r=0.5 a)=0.035$. The physical parameters of the CYCLONE case are given in Table V. Let us point out that in view of preparing turbulent runs, these neoclassical simulations were performed by retaining poloidal modes $m \in[-5,5]$ of the axisymmetric potential $\phi_{n=0}$. It is shown in Fig. 12(a) that the coarse graining procedure may stabilize the weight spreading, reflected by the saturation of the total weight variance once the system has reached collisional equilibrium. As a corollary, the numerical noise defined in Eq. (95) also stabilizes at the same time, as shown in Fig. 12(b). The neoclassical properties of the simulations are essentially unaffected by the coarse graining procedure, as shown in Fig. 13, presenting the heat diffusivity $\chi_{H i}$ as a function of the radial position for the CYCLONE case.

\section{B. Collisional $\delta f$-algorithm with local/canonical Maxwellian background switching}

Although we only considered a local Maxwellian background so far $\left(f_{0}=f_{\mathrm{LM}}\right)$, there is, in fact, some freedom in the choice of $f_{0}$, as stated in Sec. III, at least for carrying out the collisionless dynamics of global simulations represented by the left hand side of Eq. (7). The collisionless version of ORB5 usually considers a canonical Maxwellian background, defined as follows:

$$
f_{\mathrm{CM}}=\frac{\mathcal{N}\left(\Psi_{0}\right)}{\left(2 \pi \mathcal{T}\left(\Psi_{0}\right) / m\right)^{3 / 2}} \exp \left[-\frac{m v_{\|}^{2}}{2 \mathcal{T}\left(\Psi_{0}\right)}-\frac{B \mu}{\mathcal{T}\left(\Psi_{0}\right)}\right]
$$

where "temperature" $\mathcal{T}$ and "density" $\mathcal{N}$ are functions of the toroidal canonical momentum $\Psi_{0}=\Psi+q F(\Psi) v_{\|} / m B$, where $F(\Psi)=R B_{\varphi}$ is the poloidal current flux function. Note that due to the velocity dependence of $\Psi_{0}, \mathcal{N}$ and $\mathcal{T}$ differ from the actual density $n_{0}$ and temperature $T_{0}$ associated with $f_{\mathrm{CM}}$. Following the procedure described in Sec. III, the equations for the evolution of the $w$ - and $p$-weights in case of global collisionless simulations considering a canonical Maxwellian background $f_{\mathrm{CM}}$ can be derived, leading to

$$
\begin{aligned}
& \frac{\mathrm{d}}{\mathrm{d} t} w_{\mathrm{CM}, r}(t)=-\left.p_{\mathrm{CM}, r}(t) \frac{1}{f_{\mathrm{CM}}} \frac{D}{D t} f_{\mathrm{CM}}\right|_{\left[z_{r}(t), t\right]}, \\
& \frac{\mathrm{d}}{\mathrm{d} t} p_{\mathrm{CM}, r}(t)=\left.p_{\mathrm{CM}, r}(t) \frac{1}{f_{\mathrm{CM}}} \frac{D}{D t} f_{\mathrm{CM}}\right|_{\left[z_{r}(t), t\right]} .
\end{aligned}
$$

When considering this latter decomposition, one issue that needs to be carefully addressed is related to the field equation. Previously, ORB5 has assumed that the background distributions themselves verify quasineutrality. ${ }^{8}$ This condition may easily be satisfied, in practice, when working with a local Maxwellian as its corresponding density is straightforward to prescribe [the field $n_{0}(\Psi)$ in Eq. (6)]. Prescribing the background density is, however, less evident when working

TABLE V. Run parameters, CYCLONE case.

\begin{tabular}{llllll}
\hline \hline Profile 3 & $a / R_{0}=0.37$ & $T_{e} / T_{i}=1$ & $\kappa_{T}=2.63$ & $\kappa_{n}=0.81$ & $1 / \rho^{*}=180$ \\
\hline
\end{tabular}



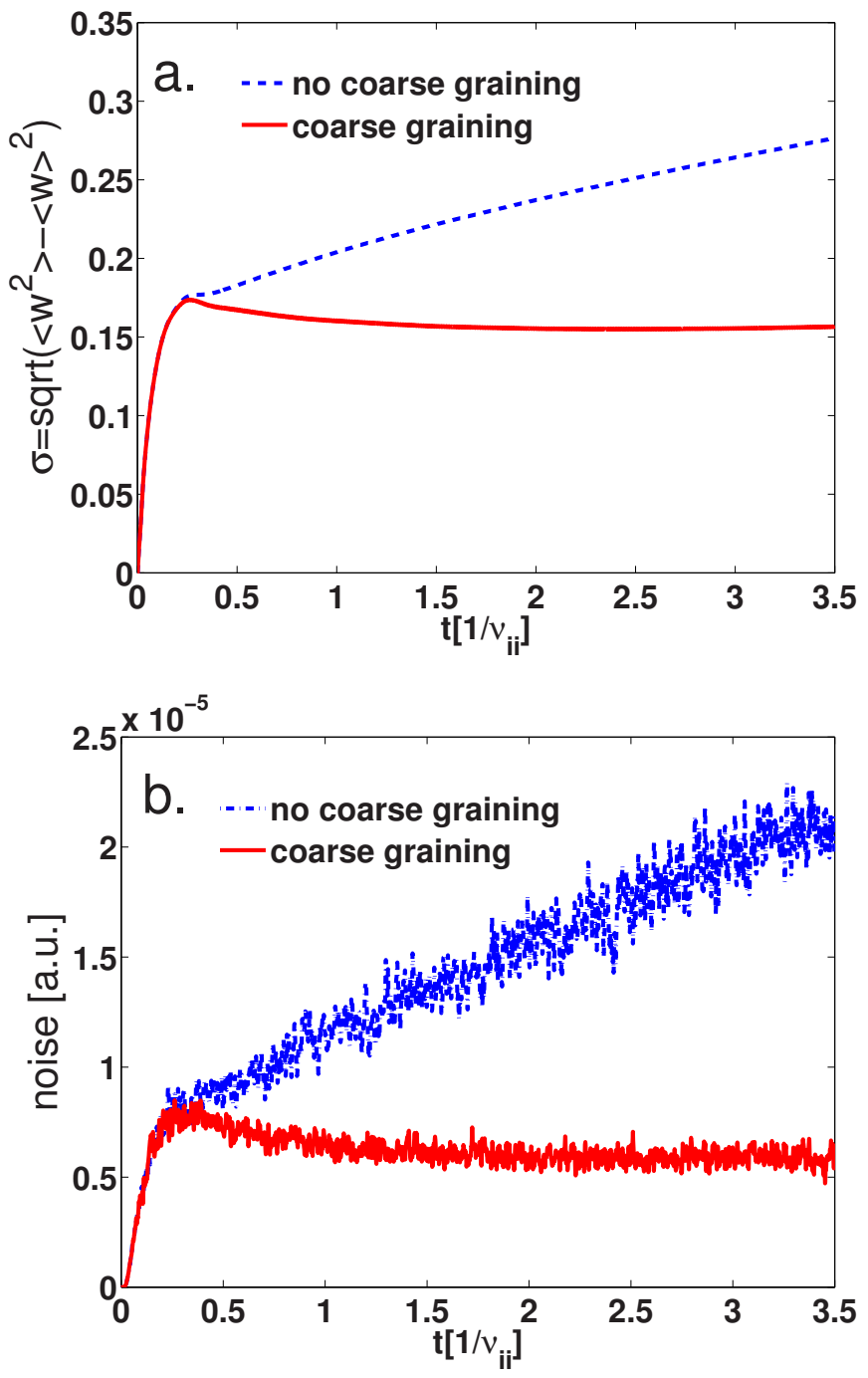

FIG. 12. (Color online) Effects of the coarse graining procedure. (a) Total weight variance and (b) numerical noise. CYCLONE parameters with $\nu^{*}(r / a=0.5)=0.035$. The noise and the weight spreading are fully stabilized by the coarse graining algorithm for this low collisionality.

with a canonical Maxwellian as the field $\mathcal{N}\left(\Psi_{0}\right)$ in Eq. (96) may not be identified to the actual background density, with $\Psi_{0}$ being a function of velocity. In this case, it is simpler to assume that it is the full initial distribution that satisfies the quasineutrality condition. We introduce the following corrected density $\overline{\delta n_{0}}$ :

$$
\begin{aligned}
\overline{\delta n_{0}}(\vec{x})= & \int \frac{B_{\|}^{*}}{m} \mathrm{~d}^{3} R \mathrm{~d} v_{\|} \mathrm{d} \mu\left[f_{i}\left(\vec{R}, v_{\|}, \mu, t=0\right)\right. \\
& \left.-f_{0, i}\left(\vec{R}, v_{\|}, \mu\right)\right] \delta(\vec{R}+\vec{\rho}-\vec{x}),
\end{aligned}
$$

which is subtracted at each time step on the RHS of the quasineutrality equation (16), leading to

$$
\begin{aligned}
& \frac{e n_{i 0}(\Psi)}{T_{e}(\Psi)}[\phi(\vec{x}, t)-\langle\phi\rangle(\Psi, t)]-\nabla_{\perp} \cdot\left[\frac{n_{i 0}(\Psi)}{B \Omega_{i}} \nabla_{\perp} \phi\right] \\
& \quad=\overline{\delta n_{i}}(\vec{x})-\overline{\delta n_{0}}(\vec{x}) .
\end{aligned}
$$

The background $f_{\mathrm{CM}}$ is a stationary state of the collisionless gyrokinetic equation. Making use of the two-weight ap-

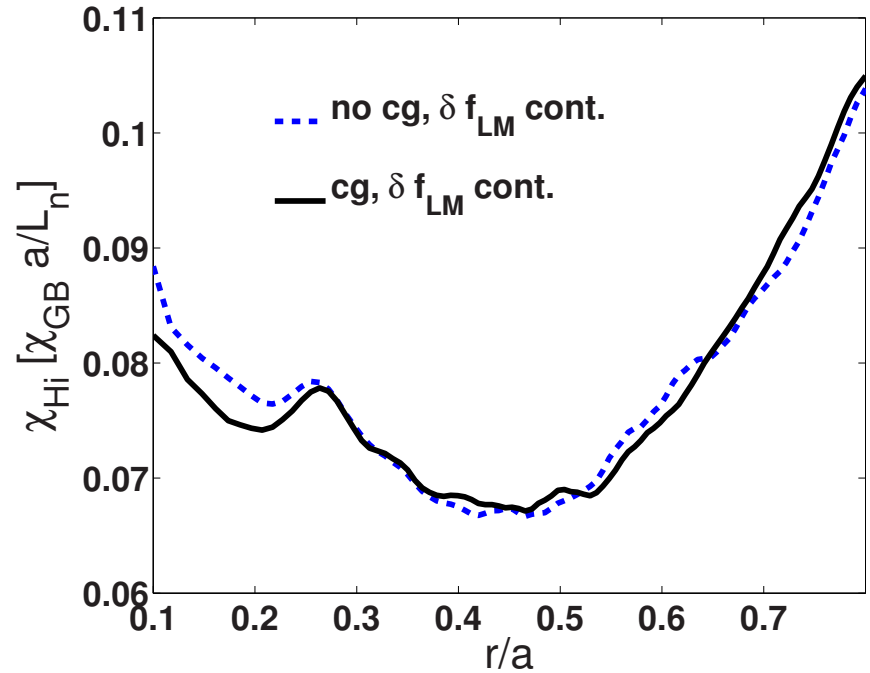

FIG. 13. (Color online) The coarse graining procedure does not affect the fundamental neoclassical results such as the heat diffusivity $\chi_{H i}$. Both simulations, with and without coarse graining, show that the transport level may be conserved while controlling the noise. $\nu^{*}(r / a=0.5)=0.035$; $t=0.5 \tau_{i i}(r / a=0.5)$.

proach, it is possible to take advantage of this collisionless equilibrium function even in collisional runs. This is achieved by switching, in the frame of the time splitting scheme, between a canonical Maxwellian background representation for carrying out the collisionless advection step and a local Maxwellian background representation for carrying out the collisional step. This mixed background approach is schematized in Fig. 14. Considering a local Maxwellian background for carrying out collisions is indeed a practical constraint as the collision operators have been linearized with respect to such a distribution. Let us point out that this mixed background scheme is essentially used for ions, as $f_{\mathrm{LM}} \simeq f_{\mathrm{CM}}$ for electrons.

For carrying out collisions, such a switching scheme requires transforming for each marker $r$ between its two weights $\left(w_{\mathrm{CM}, r}, p_{\mathrm{CM}, r}\right)$ in the canonical Maxwellian background $f_{\mathrm{CM}}$ representation and its two weights $\left(w_{\mathrm{LM}, r}, p_{\mathrm{LM}, r}\right)$ in the local Maxwellian background $f_{\mathrm{LM}}$ representation. Two equations are necessary for defining this weight transformation. The first is obtained by requiring that at any time the same total distribution $f$ must be obtained by the two representations,

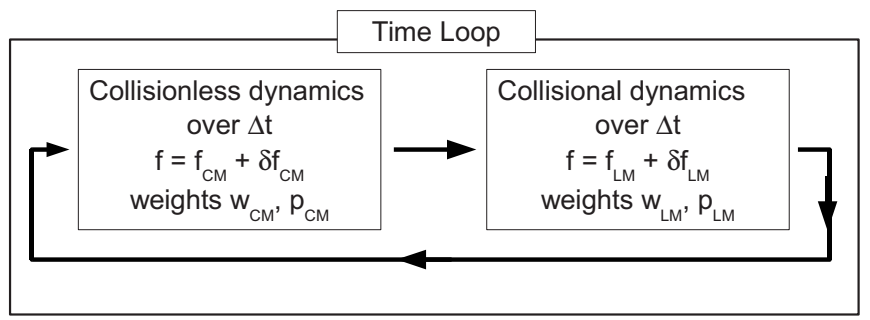

FIG. 14. Mixed background collisional delta-f algorithm: time loop for switching between a canonical Maxwellian background for stepping the collisionless dynamics and a local Maxwellian background for stepping the collisional dynamics. 


$$
f=f_{\mathrm{CM}}+\delta f_{\mathrm{CM}}=f_{\mathrm{LM}}+\delta f_{\mathrm{LM}},
$$

where $\delta f_{\mathrm{CM}}$ and $\delta f_{\mathrm{LM}}$ stand for the deviations of the full distribution from the canonical and local Maxwellian backgrounds, respectively. Together with the PIC discretizations, Eq. (101) implies for the weights of each marker

$$
w_{\mathrm{CM}, r}+p_{\mathrm{CM}, r}=w_{\mathrm{LM}, r}+p_{\mathrm{LM}, r} \text {. }
$$

The second equation is obtained by noticing that at any time and for any marker $r$, one also has

$$
\frac{p_{\mathrm{CM}, r}}{p_{\mathrm{LM}, r}}=\frac{f_{\mathrm{CM}}\left(z_{r}\right)}{f_{\mathrm{LM}}\left(z_{r}\right)}=\sigma_{r}
$$

having introduced the notation $\sigma_{r}$ for the ratio of the two backgrounds at the current marker position $z_{r}$ in the phase space. After the collisionless advection step, one can thus transform from canonical to local weights using Eqs. (102) and (103),

$$
\begin{aligned}
& p_{\mathrm{LM}, r}=\frac{p_{\mathrm{CM}, r}}{\sigma_{r}}, \\
& w_{\mathrm{LM}, r}=w_{\mathrm{CM}, r}+\left(1-\frac{1}{\sigma_{r}}\right) p_{\mathrm{CM}, r},
\end{aligned}
$$

and carry out the collisions in the frame of the local Maxwellian background representation, as described in Sec. III D. This will result for each marker in a new position $z_{r}^{*}$ in the phase space and a corresponding background ratio $\sigma_{r}^{*}=f_{\mathrm{CM}} /\left.f_{\mathrm{LM}}\right|_{z_{r}^{*}}$, as well as a first weight increment $\Delta w_{\mathrm{LM}}$ from self-collisions, given by Eq. (86). Let us recall that the second weight remains invariant, $\Delta p_{\mathrm{LM}}=0$, as a result of collisions. Transforming back to the canonical weights, one obtains the corresponding weight increments from the collisional step,

$$
\begin{aligned}
& \Delta p_{\mathrm{CM}, r}=\Delta \sigma_{r} p_{\mathrm{LM}, r}=\left(\frac{\sigma_{r}^{*}}{\sigma_{r}}-1\right) p_{\mathrm{CM}, r}, \\
& \Delta w_{\mathrm{CM}, r}=\Delta w_{\mathrm{LM}, r}-\Delta p_{\mathrm{CM}, r}=\Delta w_{\mathrm{LM}, r}-\left(\frac{\sigma_{r}^{*}}{\sigma_{r}}-1\right) p_{\mathrm{LM}, r} .
\end{aligned}
$$

The new phase-space positions $z_{r}^{*}$, as well as the weights $w_{\mathrm{CM}, r}^{*}=w_{\mathrm{CM}, r}+\Delta w_{\mathrm{CM}, r}$ and $p_{\mathrm{CM}, r}^{*}=p_{\mathrm{CM}, r}+\Delta p_{\mathrm{CM}, r}$ $=p_{\mathrm{CM}, r} \sigma_{r}^{*} / \sigma_{r}$ of each marker, may then be used for carrying out the next collisionless advection step, thus closing the time loop in Fig. 14.

In order to validate the background switching algorithm, a collisional simulation is carried out with this new scheme and compared to the results obtained for the same physical conditions with the standard fixed local Maxwellian background approach. Both algorithms make use of the coarse graining procedure described in Sec. V A. The simulations solve the global neoclassical problem and consider the physical system described in Table $\mathrm{V}$ with $\nu^{*}(r=0.5 a)=0.35$. The two simulations must, in particular, represent identical initial
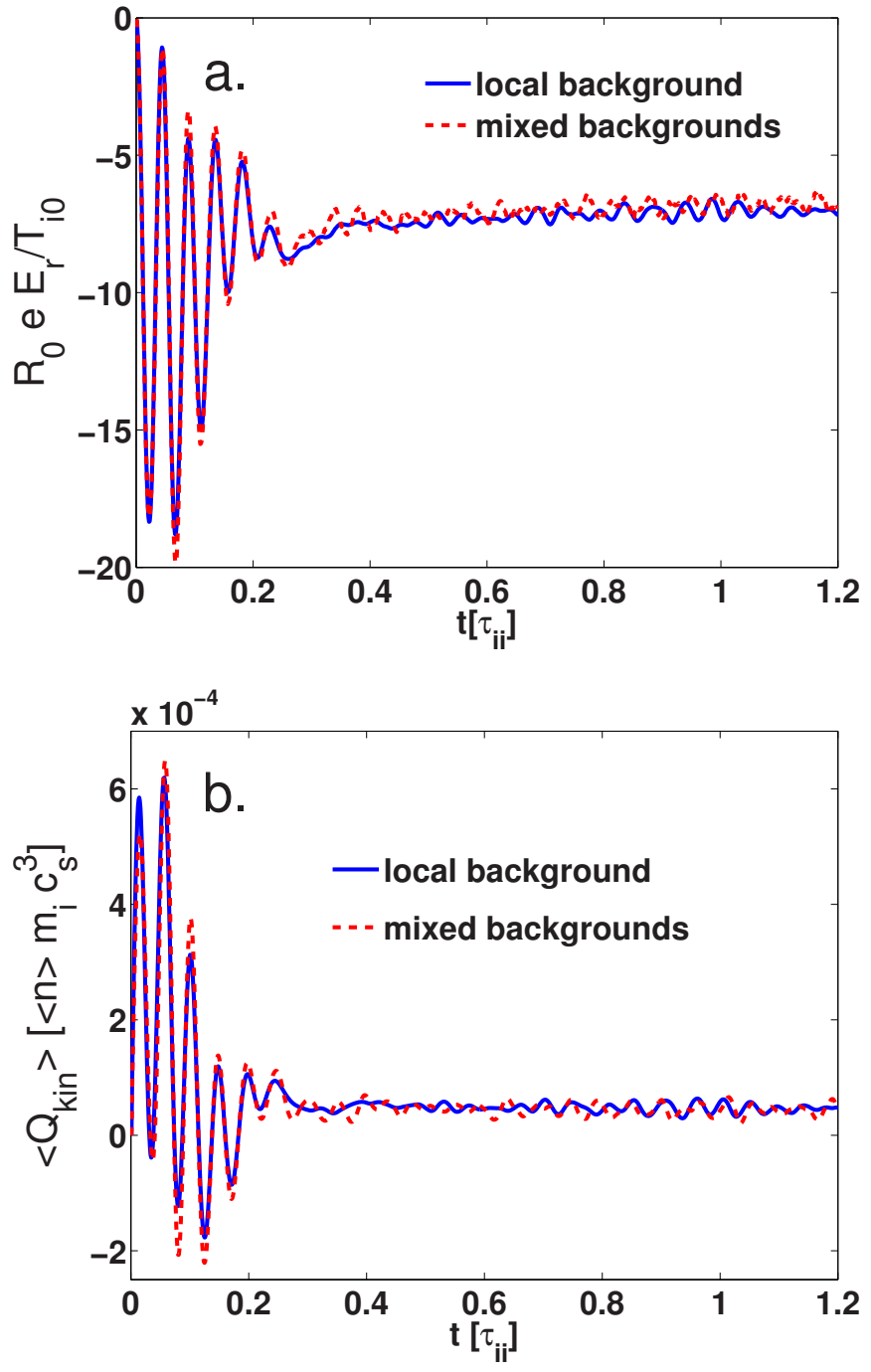

FIG. 15. (Color online) Comparing the fixed local Maxwellian background approach with the novel collisional algorithm switching between a canonical and a local Maxwellian background. (a) Radial electric field $E_{r}$ and (b) ion kinetic energy flux $Q_{\text {kin }}$ as a function of time. $\nu^{*}=0.35$.

conditions, chosen as the local Maxwellian for the total initial distribution, $f(t=0)=f_{\mathrm{LM}}$. The standard algorithm is thus carried out with the local Maxwellian as a fixed background $f_{0}=f_{\mathrm{LM}}$ and $\delta f_{\mathrm{LM}}(t=0)=0$, while the new algorithm starts from the canonical Maxwellian background $f_{0}=f_{\mathrm{CM}}$ and $\delta f_{\mathrm{CM}}(t=0)=f_{\mathrm{LM}}-f_{\mathrm{CM}}$, but then switches to the local Maxwellian background representation for carrying out collisions.

Considering the already mentioned assumption that initial distribution density satisfies the quasineutrality condition, both algorithms (fixed and switching background) should be identical in the limit of sufficient resolution. This is confirmed by the results shown in Fig. 15 for the test case problem considered here. Figure 15(a) plots the time evolution of the radial field at $r / a=0.5$ toward its equilibrium state over a typical collision time, while Fig. 15(b) presents the time traces for the ion kinetic energy flux. Note that the curves related to the two numerical approaches are indeed essentially identical.

One of the advantages of using a canonical Maxwellian 

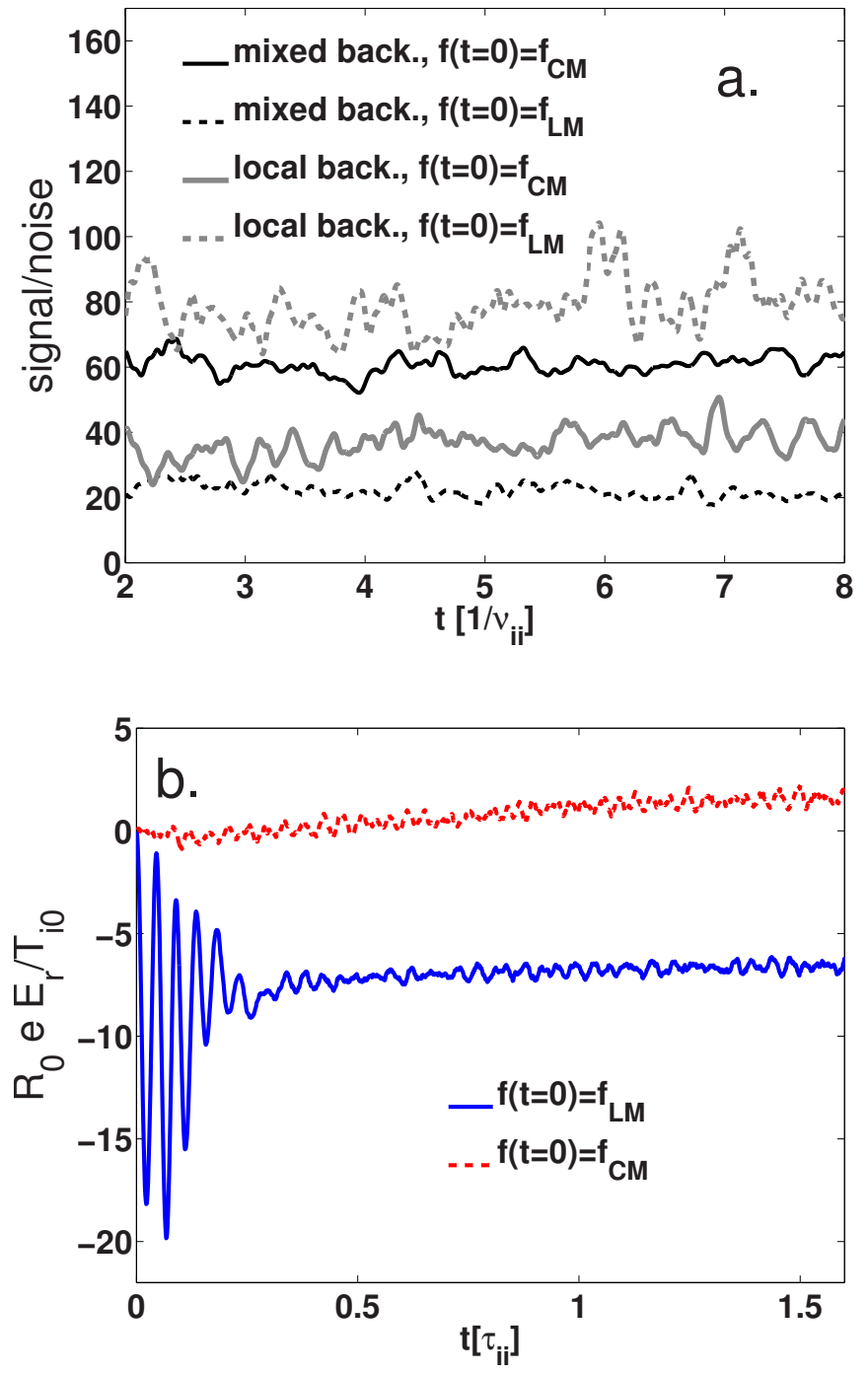

FIG. 16. (Color online) Results from global neoclassical simulations using the mixed background approach and the standard local background method, considering different initial conditions: both a local Maxwellian $f_{\mathrm{LM}}$ and a canonical Maxwellian $f_{\mathrm{CM}}$. (a) Signal/noise ratio, $\nu^{*}(r / a=0.5)=0.035$ and (b) radial electric field $E_{r}$ at $r / a=0.5, \nu^{*}=0.35$.

background for carrying out the collisionless dynamics is the fact that significantly larger time steps, typically by a factor of 2, may be taken, than when considering a local Maxwellian background, while still ensuring a stable and equivalently accurate simulation. This is obviously related to the fact that the right hand side of the weight equation contains a term proportional to $\mathrm{d} \Psi /\left.\mathrm{d} t\right|_{0} \partial f_{\mathrm{LM}} / \partial \Psi$ in the case of a local Maxwellian background, while the corresponding term $\mathrm{d} \Psi_{0} /\left.\mathrm{d} t\right|_{0} \partial f_{\mathrm{CM}} / \partial \Psi_{0}$ in the case of a canonical Maxwellian is absent, as $\mathrm{d} \Psi_{0} /\left.\mathrm{d} t\right|_{0}=0$. Here, $\mathrm{d} /\left.\mathrm{d} t\right|_{0}$ stands for the time derivative along unperturbed trajectories, so that, in particular,

$$
\left.\frac{\mathrm{d} \Psi}{\mathrm{d} t}\right|_{0}=\left.\frac{\mathrm{d} \vec{R}}{\mathrm{~d} t}\right|_{0} \cdot \vec{\nabla} \Psi=\left(\vec{v}_{\nabla B}+\vec{v}_{c}\right) \cdot \vec{\nabla} \Psi .
$$

Considering the same basic parameters as for the simulation results of Fig. 15, Fig. 16 illustrates how the collisional algorithm based on background switching may be of an advantage when considering an appropriate initial distribution. Starting the run with $f(t=0)=f_{\mathrm{CM}}$, the algorithm with back-
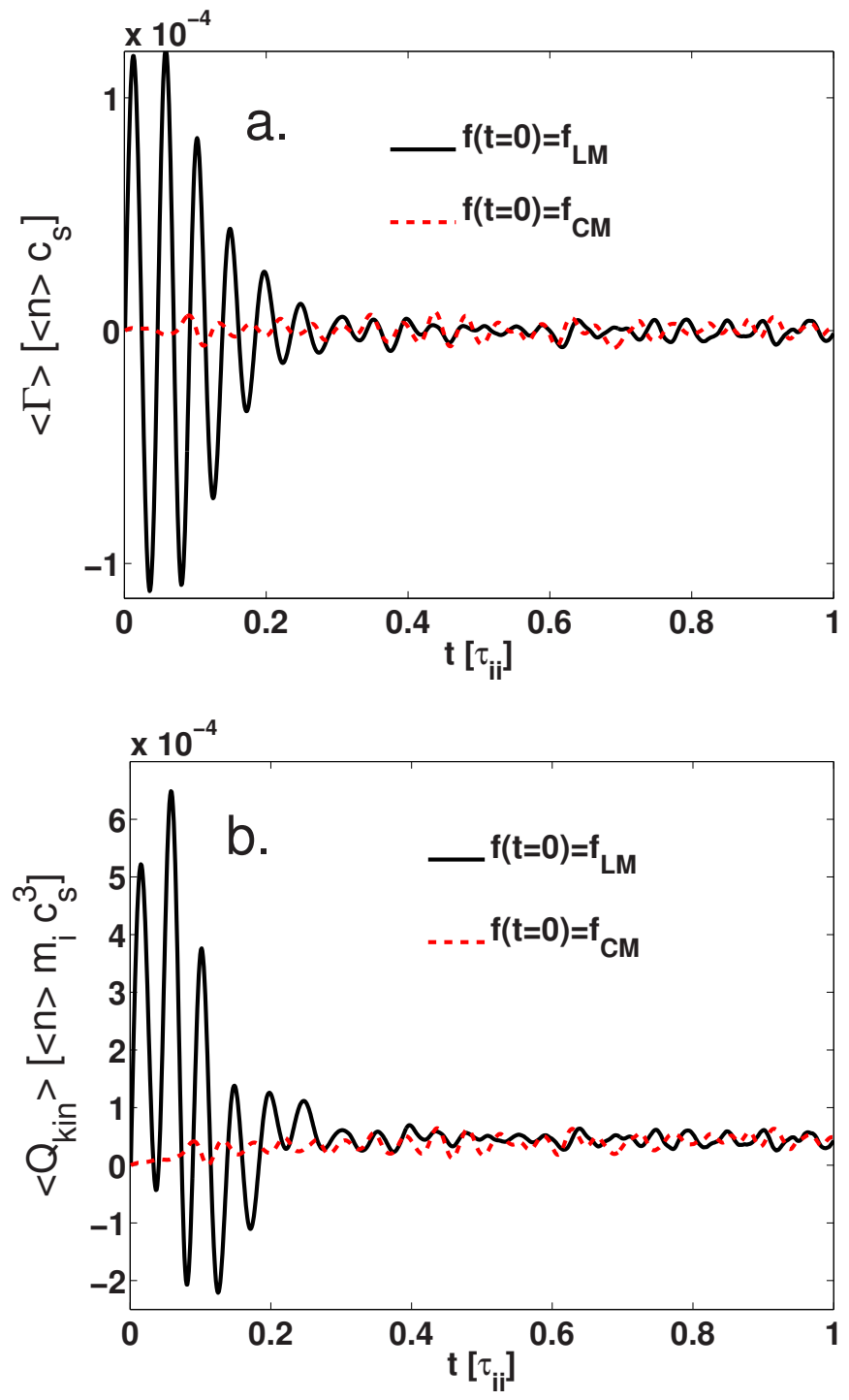

FIG. 17. (Color online) Results from global neoclassical simulations using the mixed background approach for different initial conditions. (a) Particle flux $\Gamma$ and (b) kinetic energy flux $Q_{\text {kin }}, \nu^{*}=0.35$.

ground switching provides a better signal/noise ratio than the standard fixed local Maxwellian background scheme for the same initialization, as shown in Fig. 16(a), in addition to the increased time step already mentioned. The standard algorithm is, however, less noisy than the switching background scheme when considering a local Maxwellian $f_{\mathrm{LM}}$ as the total initial distribution: $f(t=0)=f_{\mathrm{LM}}$. Figure 16(b) shows how different neoclassical radial electric fields establish themselves for the different considered initial conditions. This is consistent with the fact that different toroidal flows are generated for the two types of initial distributions, which lead to different radial electric fields, in agreement with the force balance relation (50). In fact, the radial electric fields have opposite signs, depending on whether the simulation starts from a canonical or local Maxwellian. Note, in particular, the absence of GAM oscillations in Fig. 16(b) for the initialization $f(t=0)=f_{\mathrm{CM}}$. The particle and the ion kinetic energy fluxes, however, appear not to be sensitive to the initial conditions, as shown in Fig. 17. 


\section{CONCLUSION}

Collisional effects have been added to the global gyrokinetic $\delta f$ particle-in-cell code ORB5 in the form of electronion pitch angle scattering, as well as electron-electron and ion-ion linearized self-collisions. The corresponding collision operators have been implemented using a robust algorithm based on a Langevin-type approach, which addresses the possible issue of overshooting the pitch angle domain as a result of finite time stepping.

Using these newly implemented collision operators, neoclassical transport in a tokamak has been investigated. Simulations have been carried out both within the standard neoclassical approximation as well as beyond, amounting to, respectively, neglecting or keeping drifts in the marker trajectories. Contrary to the former simulations, which are local to a magnetic surface, the latter, including the full particle trajectories, need to be carried out over the whole plasma volume and are therefore global. Global simulations have also been carried out, accounting for the possible significant effects of self-consistent electrostatic fields.

Successful benchmarks of the collisional ORB5 code were carried out by comparing the simulations both against analytical results within the standard neoclassical approximation as well as simulation results from other codes. Comparisons with the GT5D and FORTEC-3D codes were particularly valuable for validating the global simulations.

In agreement with the previous studies, limitations of the standard neoclassical approximations have been pointed out. This includes the breakdown of neoclassical ordering near the magnetic axis, leading to reduced fluxes in this region compared to those predicted by the standard theory. The role played by the poloidally asymmetric modes of the electrostatic potential, usually neglected in neoclassical transport studies, has also been analyzed.

Finally, the crucial issue of numerical noise, common to all PIC simulations, was addressed. The numerical noise problem is exacerbated in collisional simulations based on the Langevin approach as a result of the so-called weight spreading effect. To this end, a novel $\delta f$-algorithm, which switches between a canonical and a local Maxwellian background for, respectively, carrying out the collisionless and collisional dynamics in the frame of a time splitting scheme, has been developed. Only temporarily during the collision step does one switch to a local Maxwellian background, around which the collision operators have been conveniently linearized. This novel $\delta f$-algorithm was carefully benchmarked against the standard fixed local Maxwellian background approach, and its beneficial effect on reducing weights and enabling increased time steps, by as much as a factor of 2, has been illustrated. Combined with a coarse graining procedure, newly implemented in the ORB5 code as well, this novel algorithm enables to obtain neoclassical equilibria with a good signal/noise ratio. Such global neoclassical equilibria, including self-consistent background electric fields, provide a sound starting point for carrying out turbulent collisional gyrokinetic simulations, which shall be the focus of our ongoing research effort.

\section{ACKNOWLEDGMENTS}

Simulations were performed on the Monte Rosa CRAY XT-5 supercomputer of the Swiss National Supercomputing Center, the HPC-FF cluster of the Jülich Forschungszentrum, and the BlueGene/P supercomputer of the CADMOS project. This work was partly supported by the Swiss National Science Foundation. The authors would like to thank S. Satake and Y. Idomura for supporting the benchmarking efforts by providing their own neoclassical data. The authors would furthermore like to thank O. Sauter for the fruitful discussions related to the neoclassical transport.

${ }^{1}$ J. A. Krommes, Phys. Plasmas 6, 1477 (1999).

${ }^{2}$ S. Jolliet, B. F. McMillan, T. Vernay, L. Villard, A. Bottino, and P. Angelino, Phys. Plasmas 16, 052307 (2009).

${ }^{3}$ J. Candy and R. E. Waltz, Phys. Plasmas 13, 032310 (2006).

${ }^{4}$ B. F. McMillan, S. Jolliet, T. M. Tran, L. Villard, A. Bottino, and P. Angelino, Phys. Plasmas 15, 052308 (2008).

${ }^{5}$ A. A. Schekochihin, S. C. Cowley, W. Dorland, G. W. Hammett, G. G. Howes, G. G. Plunk, E. Quataert, and T. Tatsuno, Plasma Phys. Controlled Fusion 50, 124024 (2008).

${ }^{6}$ Z. Lin, T. S. Hahm, W. W. Lee, W. M. Tang, and R. B. White, Science 281, 1835 (1998).

${ }^{7}$ Z. Lin, T. S. Hahm, W. W. Lee, W. M. Tang, and P. H. Diamond, Phys. Rev. Lett. 83, 3645 (1999).

${ }^{8}$ S. Jolliet, A. Bottino, P. Angelino, R. Hatzky, T. M. Tran, B. F. McMillan, O. Sauter, K. Appert, Y. Idomura, and L. Villard, Comput. Phys. Commun. 177, 409 (2007).

${ }^{9}$ X. Q. Xu and M. N. Rosenbluth, Phys. Fluids B 3, 627 (1991).

${ }^{10}$ A. M. Dimits and B. I. Cohen, Phys. Rev. E 49, 709 (1994).

${ }^{11}$ Y. Chen and R. B. White, Phys. Plasmas 4, 3591 (1997).

${ }^{12}$ Z. Lin, W. M. Tang, and W. W. Lee, Phys. Plasmas 2, 2975 (1995).

${ }^{13}$ S. Brunner, E. Valeo, and J. A. Krommes, Phys. Plasmas 6, 4504 (1999).

${ }^{14}$ Y. Chen and S. E. Parker, Phys. Plasmas 14, 082301 (2007).

${ }^{15}$ G. Hu and J. A. Krommes, Phys. Plasmas 1, 863 (1994).

${ }^{16}$ O. Sauter, C. Angioni, and Y. R. Lin-Liu, Phys. Plasmas 6, 2834 (1999).

${ }^{17}$ C. Angioni and O. Sauter, Phys. Plasmas 7, 1224 (2000).

${ }^{18}$ E. A. Belli and J. Candy, Theory of Fusion Plasmas. Joint VarennaLausanne International Workshop, AIP Conf. Proc. No. 1069, (2008), p. 15.

${ }^{19}$ W. X. Wang, N. Nakajima, M. Okamoto, and S. Murakami, Plasma Phys. Controlled Fusion 41, 1091 (1999).

${ }^{20}$ W. X. Wang, F. L. Hinton, and S. K. Wong, Phys. Rev. Lett. 87, 055002 (2001).

${ }^{21}$ W. X. Wang, G. Rewoldt, W. M. Tang, F. L. Hinton, J. Manickam, L. E. Zakharov, R. B. White, and S. Kaye, Phys. Plasmas 13, 082501 (2006).

${ }^{22}$ Y. Idomura, H. Urano, N. Aiba, and S. Tokuda, Nucl. Fusion 49, 065029 (2009).

${ }^{23}$ X. Garbet, G. Dif-Pradalier, C. Nguyen, Y. Sarazin, V. Grandgirard, and Ph. Ghendrih, Phys. Plasmas 16, 062503 (2009).

${ }^{24}$ T. S. Hahm, Phys. Fluids 31, 2670 (1988).

${ }^{25}$ A. M. Dimits, L. L. LoDestro, and D. H. E. Dubin, Phys. Fluids B 4, 274 (1992).

${ }^{26}$ P. Helander, Phys. Plasmas 7, 2878 (2000).

${ }^{27}$ I. G. Abel, M. Barnes, S. C. Cowley, W. Dorland, and A. A. Schekochihin, Phys. Plasmas 15, 122509 (2008).

${ }^{28}$ A. J. Brizard, J. Decker, Y. Peysson, and F.-X. Duthoit, Phys. Plasmas 16, 102304 (2009).

${ }^{29}$ X. Lapillonne, S. Brunner, T. Dannert, S. Jolliet, A. Marinoni, L. Villard, T. Görler, F. Jenko, and F. Merz, Phys. Plasmas 16, 032308 (2009).

${ }^{30}$ P. Helander, Plasma Phys. Controlled Fusion 37, 57 (1995).

${ }^{31}$ F. L. Hinton and R. D. Hazeltine, Rev. Mod. Phys. 48, 239 (1976).

${ }^{32}$ S. Satake, R. Kanno, and H. Sugama, J. Plasma Fusion Res. 3, S1062 (2008).

${ }^{33}$ N. Winsor, J. L. Johnson, and J. M. Dawson, Phys. Fluids 11, 2448 (1968).

${ }^{34}$ M. N. Rosenbluth and F. L. Hinton, Phys. Rev. Lett. 80, 724 (1998).

${ }^{35}$ H. Sugama and T.-H. Watanabe, J. Plasma Phys. 72, 825 (2006).

${ }^{36}$ K. Itoh, K. Hallatschek, and S.-I. Itoh, Plasma Phys. Controlled Fusion 47, 451 (2005).

${ }^{37}$ F. L. Hinton and M. N. Rosenbluth, Plasma Phys. Controlled Fusion 41, 
A653 (1999).

${ }^{38}$ S. Satake, Y. Idomura, H. Sugama, and T. H. Watanabe, Comput. Phys. Commun. 181, 1069 (2010).

${ }^{39}$ C. S. Chang and F. L. Hinton, Phys. Fluids 25, 1493 (1982).

${ }^{40}$ S. P. Hirshman and D. J. Sigmar, Nucl. Fusion 21, 001079 (1981).

${ }^{41}$ K. C. Shaing and R. D. Hazeltine, Phys. Fluids B 4, 2547 (1992).

${ }^{42}$ Z. Lin, W. M. Tang, and W. W. Lee, Phys. Plasmas 4, 1707 (1997).

${ }^{43}$ S. Satake, M. Okamoto, and H. Sugama, Phys. Plasmas 9, 3946 (2002).
${ }^{44}$ A. Bergmann, A. G. Peeters, and S. D. Pinches, Phys. Plasmas 8, 5192 (2001).

${ }^{45}$ S. Jolliet, Ph.D. thesis, Ecole Polytechnique Fédérale de Lausanne, 2009. ${ }^{46}$ A. M. Dimits, G. Bateman, M. A. Beer, B. I. Cohen, W. Dorland, G. W. Hammett, C. Kim, J. E. Kinsey, M. Kotschenreuther, A. H. Kritz, L. L. Lao, J. Mandrekas, W. M. Nevins, S. E. Parker, A. J. Redd, D. E. Shumaker, R. Sydora, and J. Weiland, Phys. Plasmas 7, 969 (2000). 